This item is the archived peer-reviewed author-version of:

\title{
An evaluation of DEVS simulation tools
}

\section{Reference:}

Van Tendeloo Yentl, Vangheluwe Hans.- An evaluation of DEVS simulation tools

Simulation - ISSN 0037-5497 - (2016), p. 1-37

Full text (Publishers DOI): http://dx.doi.org/doi:10.1177/0037549716678330 


\title{
An Evaluation of DEVS simulation tools
}

\author{
Journal Title \\ $\mathrm{XX}(\mathrm{X}): 1-37$ \\ (C) The Author(s) 0000 \\ Reprints and permission \\ sagepub.co.uk/journalsPermissions.nav \\ DOI: $10.1177 /$ ToBeAssigned \\ www.sagepub.com/
}

(A)AGE

Yentl Van Tendeloo ${ }^{1}$ and Hans Vangheluwe Va,2,3 $^{1,2}$

\begin{abstract}
DEVS is a popular formalism for modelling complex dynamic systems using a discrete-event abstraction. Due to its popularity, and simplicity of the simulation kernel, a number of tools have been constructed by academia and industry. But each of these tools has distinct design goals and a specific programming language implementation. Consequently, each supports a specific set of formalisms, combined with a set of features. Performance differs significantly between different tools. We provide an overview of the current state of eight different DEVS simulation tools: ADEVS, CD ++, DEVS-Suite, MS4 Me, PowerDEVS, PythonPDEVS, VLE, and $\mathrm{X}-\mathrm{S}-\mathrm{Y}$. We compare supported formalisms, compliance, features, and performance. This paper aims to help modellers on deciding which tool to use to solve their specific problem. It further aims to help tool builders, by showing the aspects of their tool that could be extended in future versions of their tool.
\end{abstract}

\section{Keywords}

DEVS, Tools, Functionality, Performance

Received: 09-Sep-2015

Revised: 25-Jul-2016

Accepted: 17-Oct-2016

\footnotetext{
${ }^{1}$ University of Antwerp, Belgium

${ }^{2}$ Flanders Make, Belgium

${ }^{3}$ McGill University, Montréal, Canada

Corresponding author:

Yentl Van Tendeloo

Department of Mathematics and Computer Science

Middelheimlaan 1

2020 Antwerpen, Belgium

Email: Yentl.VanTendeloo@uantwerpen.be
}

Prepared using sagej.cls [Version: 2015/06/09 v1.01] 


\section{Introduction}

DEVS is a popular formalism for modelling complex dynamic systems using a discrete-event abstraction. In fact, it can serve as a simulation "assembly language" to which models in other formalisms can be mapped [1]. A number of tools have been constructed by academia and industry that allow the modelling and simulation of DEVS models. Each of these tools was developed for a specific application domain, resulting in different design goals.

Since there is no common DEVS format, used by all tools, modellers are tied to their tool. Switching between tools is far from trivial, as each tool uses its own API and language for model specification. Porting models between different tools equates to rewriting the model from scratch. This makes the initial choice on which tool to use an important choice, as switching comes at an arbitrary high cost for huge models.

The problem is further aggravated by each tool supporting a different set of formalisms and features. Even worse, performance between different tools varies by orders of magnitute, depending on the domain and model. It is therefore necessary to provide an overview of the current state of different tools. Modellers get an overview which can help them to decide on which tool to use. Tool builders get a better idea of how their tool is positioned relative to similar tools.

Previous efforts in comparing different tools are mostly done briefly in "related work" sections of new contributions $[2,3]$. These comparisons only include the most related tools, and only use criteria relevant to their contribution. Others do an in-depth analysis, but only for a limited set of simulation tools, and for a single dimension. For example, there exist in-depth performance comparisons between two simulation tools $[4,5]$, or a DEVS compliance check of two tools [6]. Other survey papers compare multiple tools, but don't go in-depth, nor do they use an exhaustive set of criteria that was externally validated [7]. End-users therefore have no complete comparison between different tools, and information is scattered.

In this paper, we compare a significant number of modern simulation tools (eight in total, selected according to several criteria), using an exhaustive set of functionality criteria, a set of DEVS compliance criteria, and a detailed performance analysis. To guarantee objective criteria selection, we use existing, independently defined criteria for functionality [8], DEVS compliance [6], and performance [9]. Only well-argued deviations from these criteria are made.

We will continue by briefly introducing the four main DEVS formalisms implemented by the tools under study (Section BACKGROUND), as well as the simulation tools themselves (Section Simulation Tools). The presented tools are compared in terms of functionality (Section FunCTIONALITY) and performance (Section Performance). Section Conclusion concludes the paper.

Prepared using sagej.cls 


\section{Background}

This section briefly introduces the four DEVS formalisms used in the remainder of this paper. This list of formalisms is by no means complete.

\section{Classic DEVS}

Classic DEVS [10] is the earliest DEVS formalism, and forms the foundation for all subsequent variants. Two significant shortcomings were encountered in the literature: the lack of parallelism [11] and the static structure of systems [12]. In response to these shortcomings, different DEVS variants were created.

\section{Parallel DEVS}

Parallel DEVS [11] was introduced to solve the performance problem caused by the (artificial) select function of Classic DEVS. Parallel DEVS is widely supported today, and has replaced Classic DEVS in most tools. Parallel DEVS models can be more easily parallelized than Classic DEVS models, due to the lack of the select function. The abstract simulator [13] shows parallel potential, by triggering all transition functions simultaneously. While it is argued that this might not be the best option in some cases [14], it clearly shows the possibility for parallelism.

\section{Dynamic Structure DEVS}

Both Classic DEVS and Parallel DEVS lack support for dynamically changing models. While it is possible to emulate dynamically changing models (e.g., by putting the model structure in the state too), this introduces a significant amount of accidental complexity. Several Dynamic Structure DEVS variants were introduced to make structural changes more intuitive, removing the need for manual "tricks". Examples are DSDEVS [15, 16] and DynDEVS [17]. These formalisms provide a mapping from an extended DEVS formalism, supporting dynamic structure, to the basic DEVS structures, proving their equivalence [12]. Performing such a mapping during simulation is inefficient, so implementations frequently use shortcuts.

\section{Cell DEVS}

Cell DEVS [18] is a combination of DEVS and Cellular Automata. Cellular Automata use a discrete time base, making them increasingly resource intensive with increasing time granularity. On the other hand, DEVS uses a discrete event time base, only taking into account points in time where events are processed and exchanged. It is not optimized, however, for synchronous communication, as is the case in Cellular Automata. Cell DEVS merges these two formalisms to combine a discrete event time base with synchronous communication. A Parallel Cell DEVS [19] variant was also introduced with the advent of Parallel DEVS.

Quantization [10] can be used to prevent the propagation of insignificant state changes, thus improving performance. As the definition of "insignificant" varies from model to model, the user has to define the significance threshold manually. Using quanitization is a trade-off between accuracy and performance.

Prepared using sagej.cls 


\begin{tabular}{lcccc}
\hline & Version & Release & Language & License \\
\hline ADEVS & 2.8 .1 & 2014 & C ++ & FreeBSD \\
CD++ & $2.0-\mathrm{R} .45$ & 1999 & $\mathrm{C}++$ \& custom & (unspecified) \\
DEVS-Suite & 2.1 .0 & 2009 & Java & LGPLv2 \\
MS 4 Me & 1.5 .0 & 2015 & Java \& custom & Proprietary \\
PowerDEVS & $2.4 \mathrm{rev}$ & 2015 & C ++ & GPLv3 \\
PythonPDEVS & 2.3 & 2015 & Python & Apache-2.0 \\
VLE & 1.2 & 2014 & C ++ XML & GPLv3 \\
$X-S-Y$ & 1.0 .0 & 2012 & Python & LGPL \\
\hline
\end{tabular}

Table 1. Simulation tools under study

\section{Simulation Tools}

This section provides a brief introduction to the DEVS simulation tools under study. We have selected the tools based on the following criteria:

- Features. Due to the wide variety in simulation software, all tools support a different set of features. In our evaluation, we consider tools with an extensive feature set, but also tools with very few, but special features.

- Performance. For large scale simulations, performance is critical. While we certainly want to include highly optimized simulation tools, we also want to include tools with a potentially bad performance in case there is some gain in other dimensions (e.g., more features).

- Popularity. Finally, popularity is an important criteria due to inertia: a popular tool will still be used as long as there are not enough convincing arguments to move to an all-round beter tool. There likely is a wellargumented reason as to why the tool is popular.

A summary of our selected tools is given in Table 1. For each tool, we provide a brief introduction, an example or screenshot where appropriate, and our rationale for inclusion of this tool.

\section{ADEVS}

ADEVS [20] is a lightweight C ++ library, offering DEVS simulation. Both atomic and coupled models are written in $\mathrm{C}++$ code, which must include the ADEVS headers. Due to the extensive use of templates, the headers contain all required source code. The simulation kernel and model are compiled into a single executable, and must therefore be recompiled after every model edit.

Example As models are pure $\mathrm{C}++$ code, there is nothing to show as an example. Examples of how the API works can be found in the documentation.

Rationale Because it is lightweight and has significant potential for static optimization, ADEVS is included for its potential performance. The use of efficient algorithms [21] and results from previous performance evaluations [4, $7,22]$ indicate that it will probably be (one of) the most efficient simulation 
tools under study. It also offers some interesting features, such as simulation of hybrid systems and OpenModelica [23] bindings.

$C D++$

$\mathrm{CD}++[24]$ is a DEVS simulator written in $\mathrm{C}++$. Simulation of Cell DEVS models is its main feature, though normal DEVS models can be simulated too. DEVS models can also be coupled to Cell DEVS models. Atomic models are written in $\mathrm{C}++$ and are linked into the simulation tool. Coupled models are written in a custom syntax, which is interpreted at simulation-time. Changes to atomic models require recompilation and linking to the simulation tool. Changes to coupled models don't require any recompilation at all, as these are interpreted during simulation. The complete behaviour of Cell DEVS models is defined using the custom syntax, which is completely interpreted. A graphical modelling environment, called $\mathrm{CD}++$ Builder [2], can be used to create the models.

Note that every exchanged event has to be a floating point number. Sending complex events, such as records, requires a workaround by mapping all attributes to a seperate port.

Example An example of the custom syntax, together with a workaround for complex events, is shown in Listing 1. Normally, a car is represented as a single object, and passed as such. In $\mathrm{CD}++$, every attribute gets its own port.

Rationale $\mathrm{CD}++$ is a mature tool and is widely used in the literature for its Cell DEVS functionality [25-27]. It is included in our evaluation due to its popularity.

Remarks Several versions of CD++ exist, such as N-CD++ [24], PCD $++[19]$, and Dynamic Structure CD++ [28]. We decided to use N-CD++ in our comparison. The authors previously compared the performance between $\mathrm{N}-$ $\mathrm{CD}++$ and $\mathrm{PCD}++$, showing that $\mathrm{N}-\mathrm{CD}++$ had a lower overhead [9], and should thus be faster. $\mathrm{N}-\mathrm{CD}++$ is also the one compared to ADEVS by the authors themselves [5], making us believe that this is the most mature version of their tool.

\section{DEVS-Suite}

DEVS-Suite [29] is the successor of DEVSJava [30]. Both are implemented in Java. Its features include visualization of coupled model simulation, event injection during simulation, and simulation tracking.

Both atomic and coupled models are written in Java and are loaded into the simulation tool through introspection. Changes require recompilation of the model, but don't require any action on the simulation tool.

Example Figure 1 shows the SimView visualization of a simple model. At the left, an overview of the model is given, showing the components of the coupled model. Each atomic model can be clicked on, revealing further information about the selected model. Simulation control buttons can be seen below, combined with sliders to determine the speed of the visualization. At the right, the model is

Prepared using sagej.cls 
Listing 1: Example CD++ coupled model

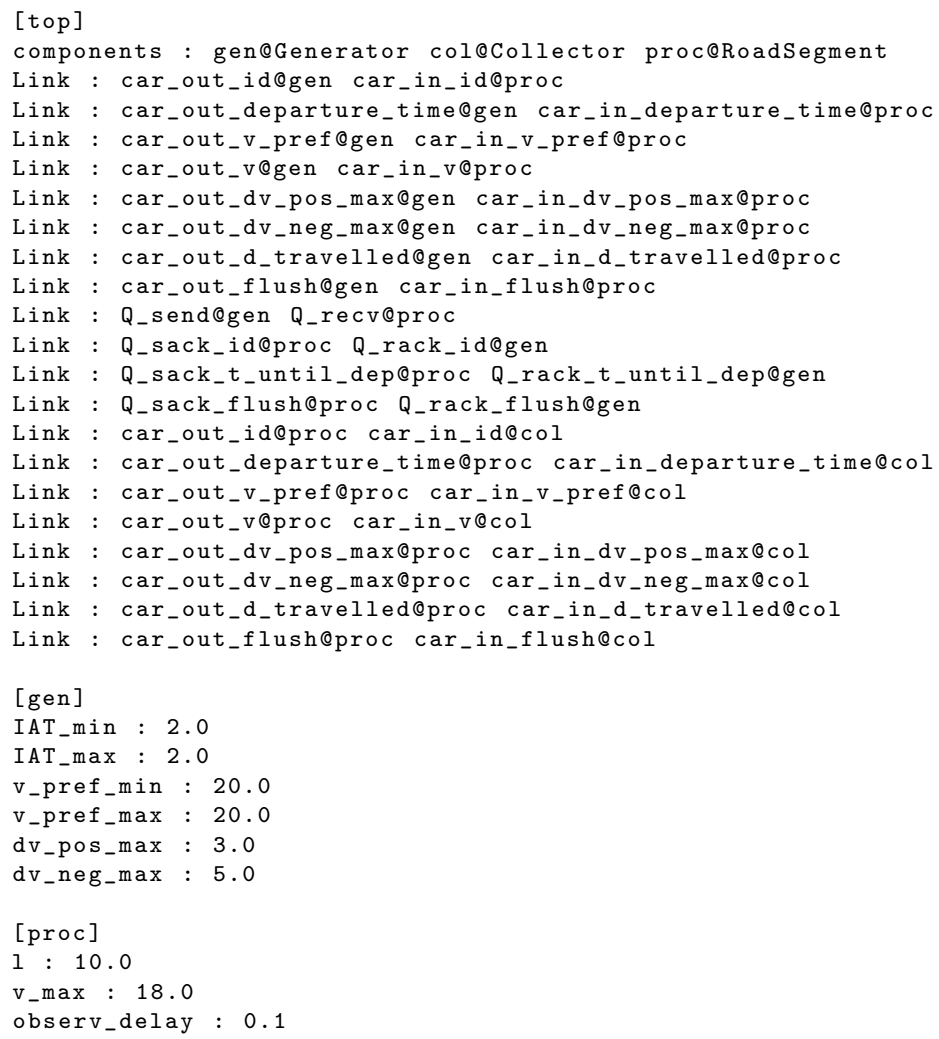

visualized, summarizing the most important information for every model, as well as its couplings. At the bottom, a console is used to print errors.

Rationale Both DEVS-Suite and DEVSJava are frequently used in the literature [31-33]. It is one of the few simulation tools which includes visualization and debugging functionality. Therefore it is added both for its popularity and features.

\section{MS4 Me}

MS4 Modeling Environment (MS4 Me) [34] is a DEVS modelling environment and simulator. It is written in Java and based on the Eclipse framework.

Atomic models are created using a custom, natural language-like language called DNL, combined with fragments of Java code. Files are automatically translated to Java code, and subsequently compiled. Coupled models can be constructed using System Entity Structure (SES) [35-37], which are pruned before simulation commences. 


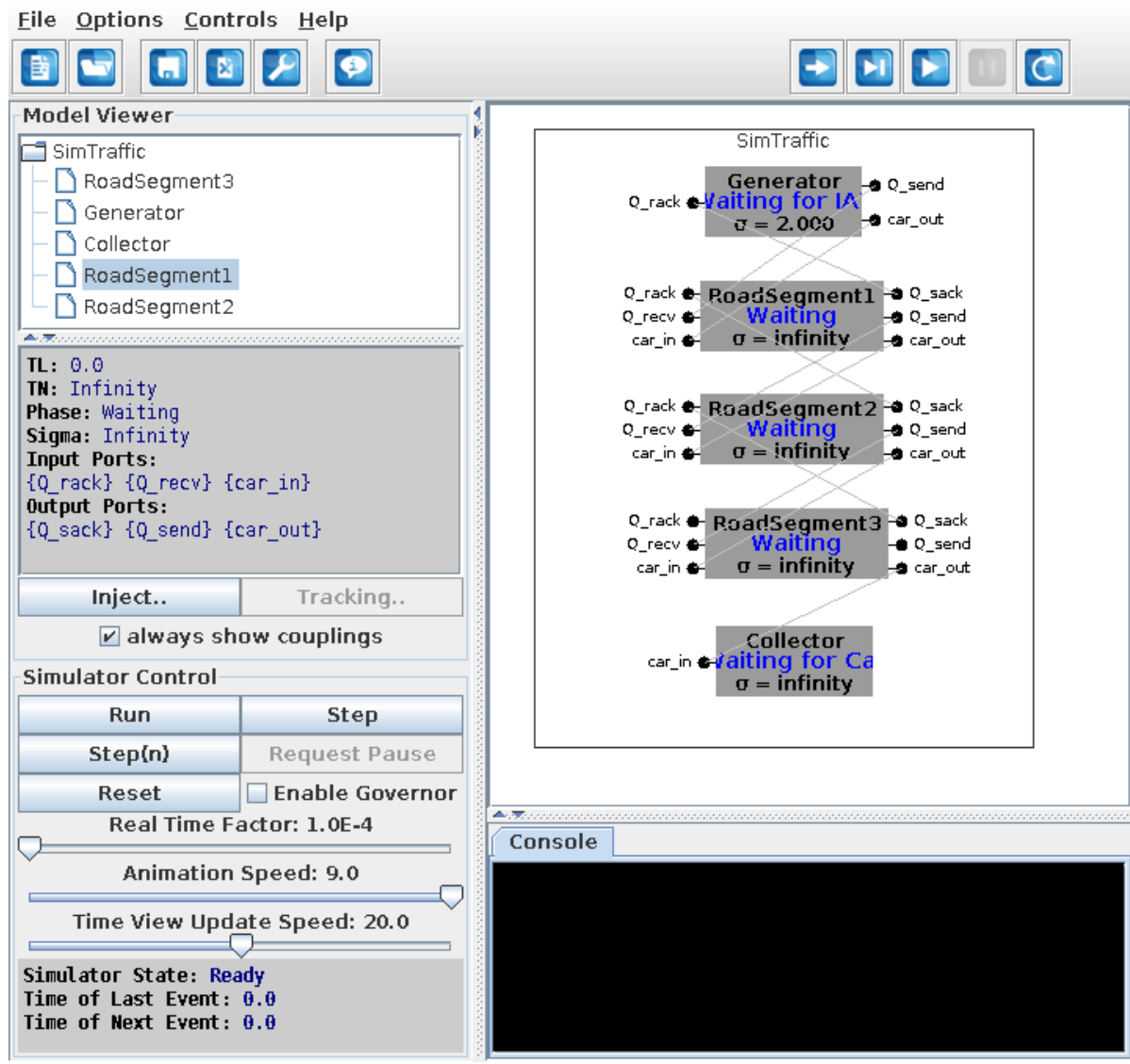

Figure 1. DEVS-Suite SimView example

Example Figure 2 presents DNL notation, used for the construction of atomic models. It shows the inclusion of Java code and the use of Java types. Figure 3 presents SES notation, used for the construction of coupled models.

Figure 4 presents the SimViewer, used for visualization of model simulation. Visualization is fairly similar to DEVS-Suite: each model can be inspected seperately, combined with a graphical representation of the complete model. The complete content of exchanged events is also visualized. It is possible to hide the couplings, the ports, or both. Simulation status, such as the current time and the number of transitions, is shown at the right. At the bottom, a console is present which logs every transition.

Rationale MS4 Me is included as it tries to move away from the use of programming languages. Whereas other tools redirect the modeller to a generalpurpose programming language for the atomic models, MS4 Me defines its own syntax to aid non-programmers. Furthermore, it is a proprietary tool, which will also give us insight in the state of the art of commercial DEVS simulation tools. 


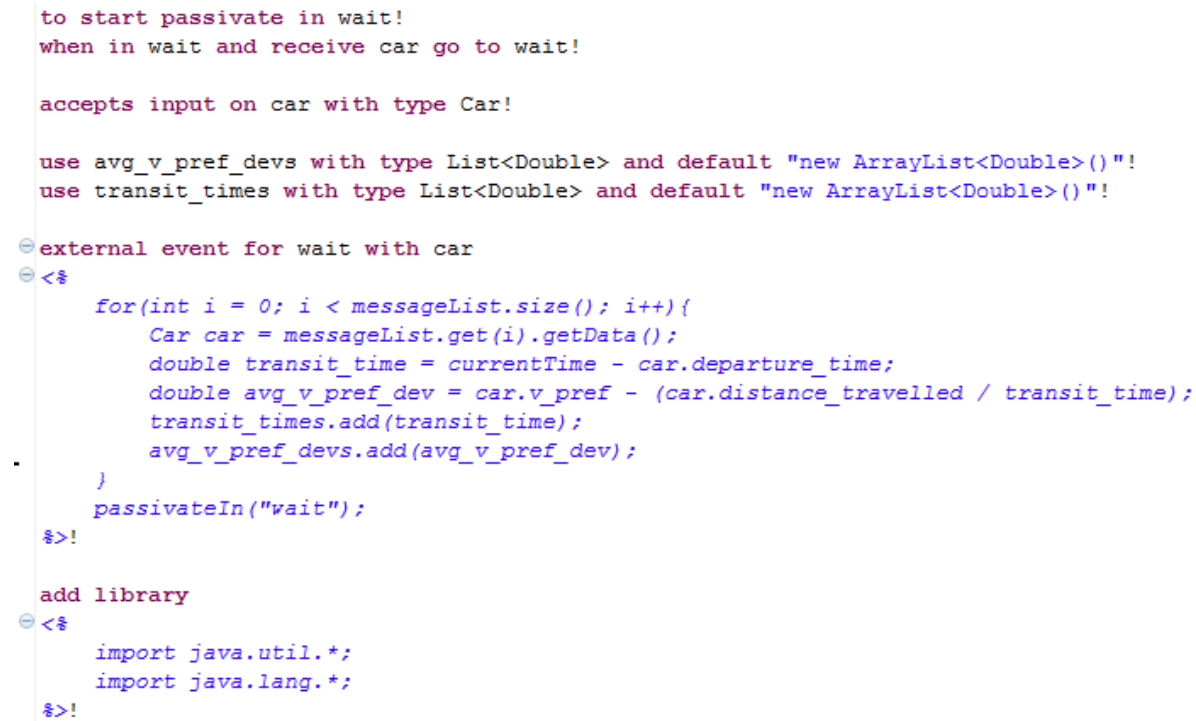

Figure 2. MS4 Me DNL example

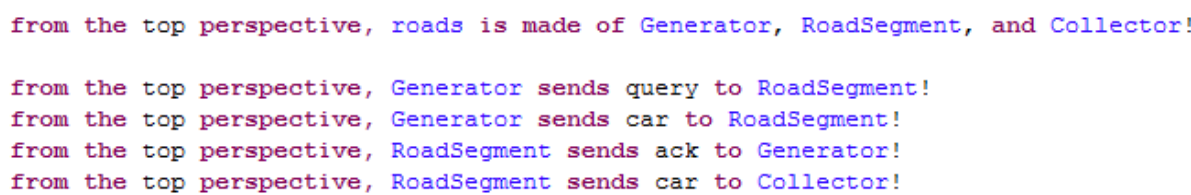

Figure 3. MS4 Me SES example

\section{PowerDEVS}

PowerDEVS [38, 39] is a Classic DEVS modelling and simulation environment implemented in $\mathrm{C}++$. It consists of a graphical modelling environment, an atomic model editor, and a code generator. The code generator generates $\mathrm{C}++$ code, which can optionally also be handwritten. PowerDEVS offers an intuitive modelling environment (with user-definable icons for models), combined with a library of models which can be reused, or used as examples.

Example Figure 5 presents the IDE, with a focus on the graphical coupling of models. The information shown is quite different from other tools, as this environment presents a design view, instead of a simulation view. In a design view, models can be constructed by adding hierarchy, adding atomic models, coupling models, or configuring simulation parameters. No information about the state of the models or the simulation is shown, as all information is independent of simulation execution. Conversely, a simulation view visualizes the state of models and previously defined hierarchy and couplings. Modifications are not allowed, as the model is already loaded and being simulated. Model simulation is done without any kind of visualization. 


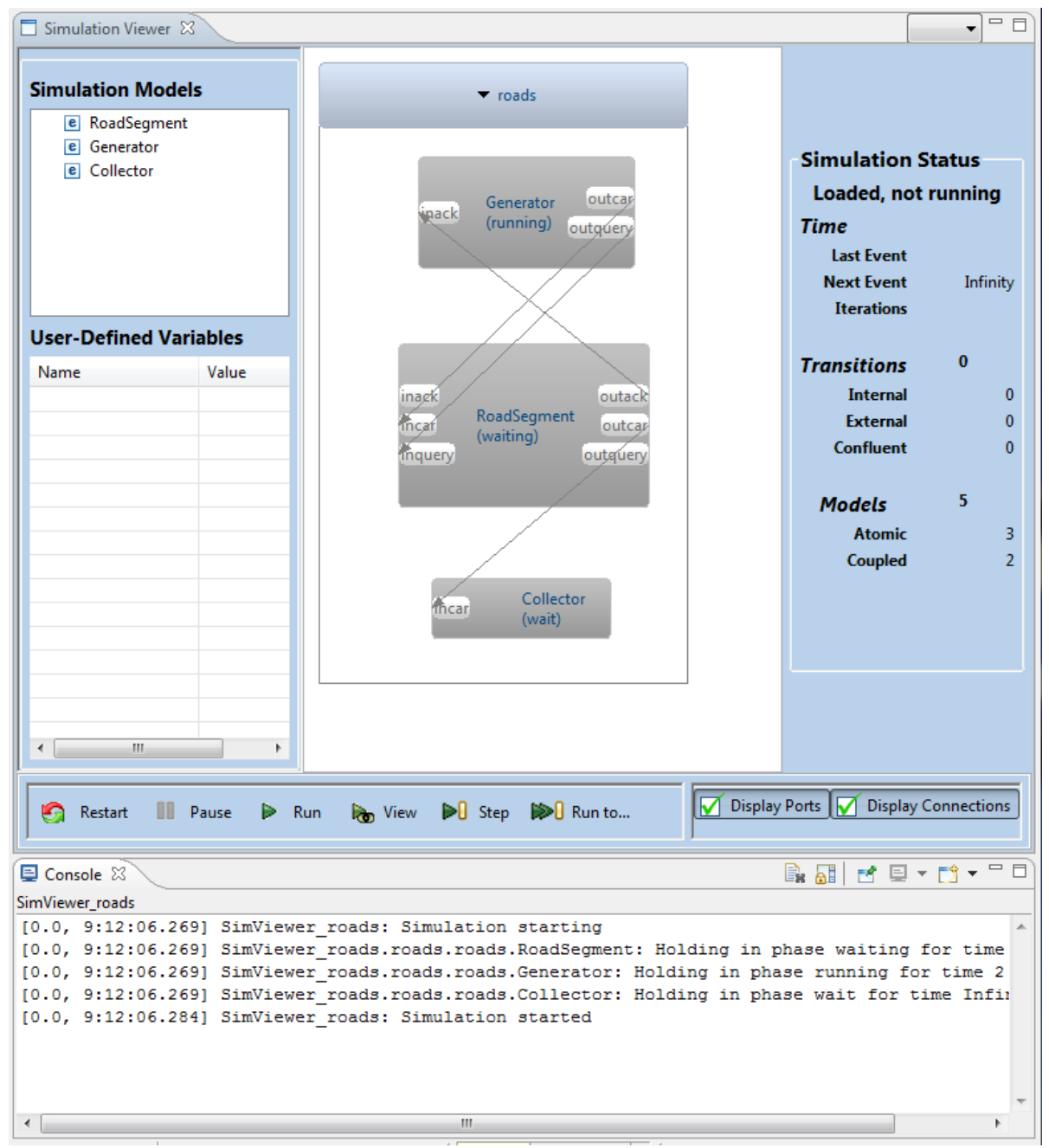

Figure 4. MS4 Me simulation example

Rationale PowerDEVS is very similar to ADEVS, as it also focusses on simulation of hybrid systems, and uses $\mathrm{C}++$ as its implementation language. Contrary to ADEVS, PowerDEVS offers a modelling environment that aids the modeller in creating valid DEVS models with a lesser degree of coding required. PowerDEVS is more aimed at non-programmers, while still offering the potential for high performance. It is thus included for both its potential performance, and its various features. 


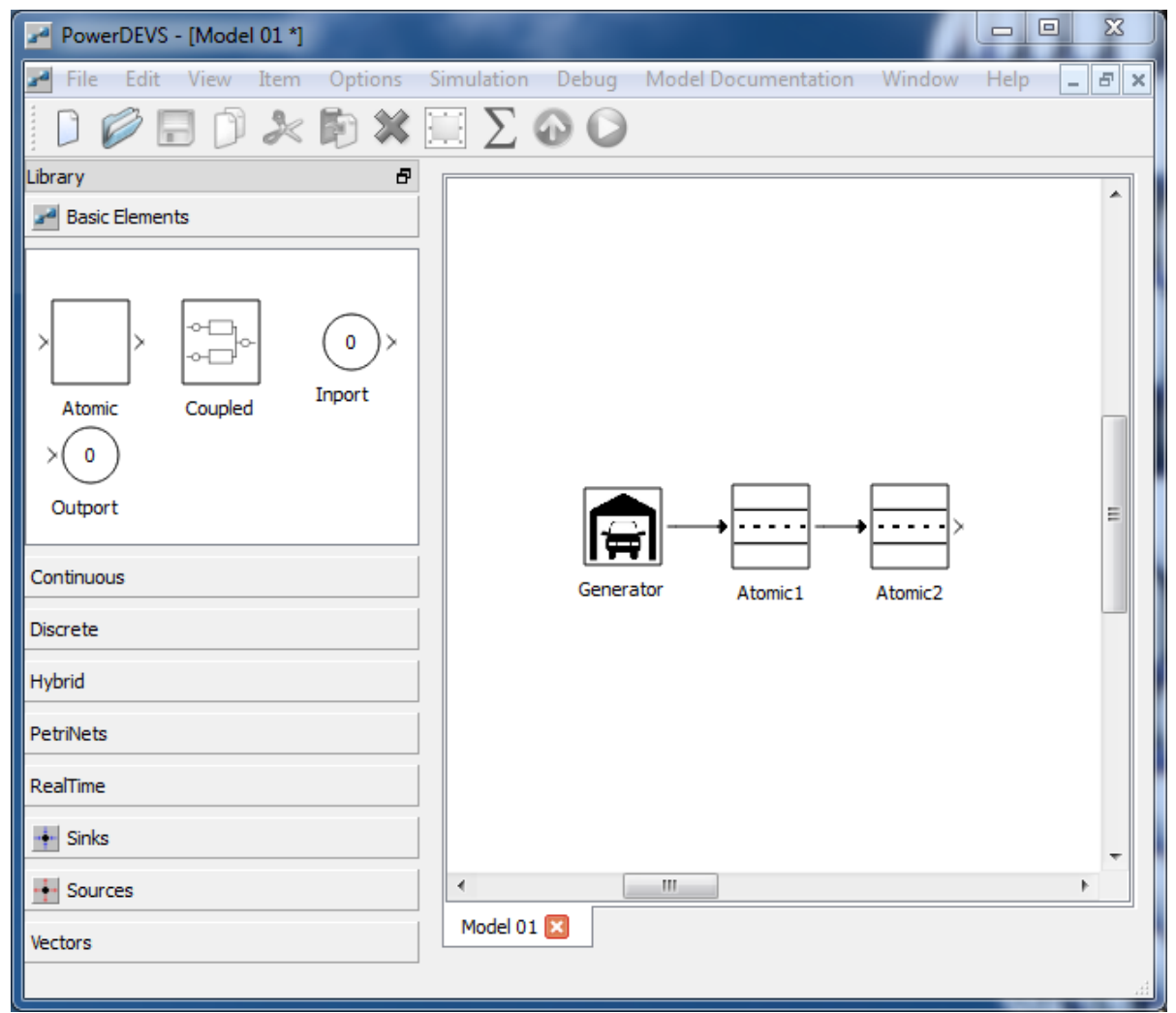

Figure 5. PowerDEVS graphical modelling environment.

\section{PythonPDEVS}

PythonPDEVS [22] is a DEVS simulator written in Python. Due to its implementation in Python, an interpreted, dynamically typed language, fast prototyping of models becomes possible. Despite its interpretation-based nature, PythonPDEVS attempts to achieve high performance. Both atomic and coupled models are written in Python, making (re)compilation unnecessary.

PythonPDEVS is used as the simulation kernel in several other tools. For example, DEVSimPy [40] offers a graphical modelling environment for coupled models, combined with an experimentation environment. A debugging frontend [41] offers a graphical modelling environment for atomic and coupled models alike, including advanced debugging capabilities.

Example As models are pure Python code, there is nothing special to show as an example. For examples of the debugging extension [41] or DEVSimPy [40], we refer to the documentation of the respective tools.

Rationale PythonPDEVS has a focus on performance, despite its implementation in an interpretation-based language $[22,42]$. We therefore included 
PythonPDEVS for its potential performance, but also for its set of supported features.

\section{VLE}

The Virtual Laboratory Environment (VLE) [43] is a multi-modelling and simulation platform written in $\mathrm{C}++$. It includes an IDE for model development and experimentation. Models are combined in "projects", which are managed by an automatically created CMake script.

Atomic models are written in $\mathrm{C}++$ and thus require recompilation of the models after changes. The simulation kernel and IDE do not need to be recompiled. Coupled models are created using either the graphical environment (called GVLE), or by manually writing the XML files.

VLE is the simulation kernel, with several bindings and "apps" to add functionality, such as an IDE (GVLE), distributed simulation using MPI (MVLE), Python bindings (PyVLE), and R bindings (RVLE).

Example Similar to PowerDEVS, VLE only offers a modelling environment without a simulation view. This environment is provided by GVLE, shown in Figure 6 .

Rationale VLE comes close to PowerDEVS in terms of supported features, as both offer a full modelling interface which generates a model for simulation by a (seperate) simulator. Its use of $\mathrm{C}++$ might also indicate that performance is one of the concerns to the developers.

\section{$X-S-Y$}

$\mathrm{X}-\mathrm{S}-\mathrm{Y}$ [44] is a DEVS simulator written in Python. Its distinguishing feature is the verification of FD-DEVS (Finite and Deterministic DEVS) models. A small command line interface is provided, allowing for simulation control.

Example As models are pure Python code, there is nothing special to show as an example.

Rationale X-S-Y offers the unique feature of supporting verification of FD-DEVS models. As no other tools implement verification, it is interesting to see how it compares to them.

Remarks During our analysis, we found that X-S-Y uses only about $10-15 \%$ of the available CPU time. Inspection of the source code revealed that a sleep of $1 \mathrm{~ms}$ occurs after every simulation step. This sleep was removed for our performance benchmarks, as it offers a fairer comparison between the used simulation algorithms.

\section{Functionality}

In this section, we will compare the functionality of the previously mentioned tools. Tools are first evaluated based on generic simulation tool criteria [8].

Prepared using sagej.cls 


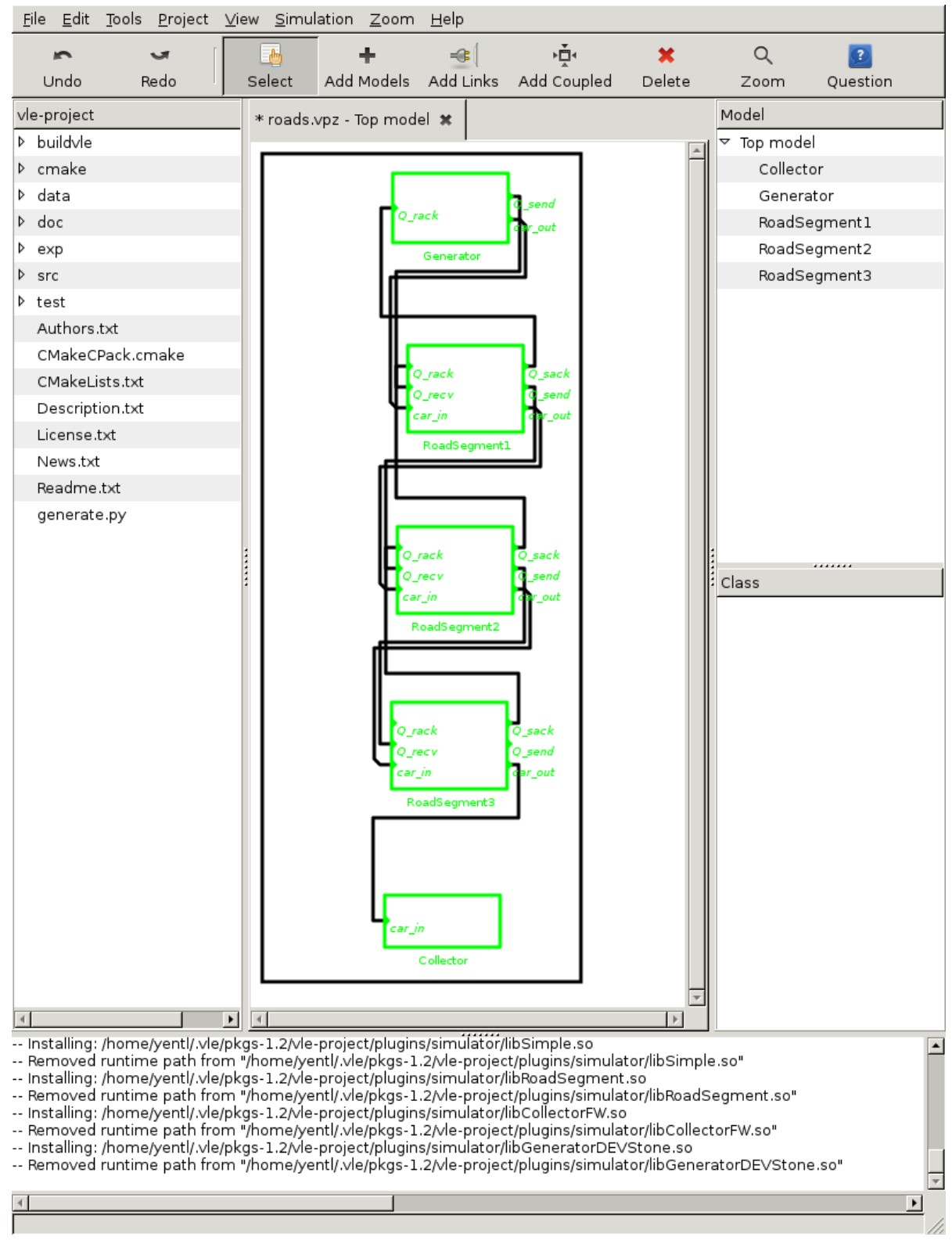

Figure 6. GVLE visualization of a coupled model 
Afterwards, a brief comparison is made based on some DEVS-specific criteria: which formalisms do they support, and how compliant are to they to them.

\section{General evaluation criteria}

The simulation tool evaluation criteria from [8] are used as a basis. Some criteria were dropped, as they are irrelevant for a DEVS-specific comparison. Others are added, in case they are deemed necessary to achieve a better distinction between the different tools.

Table 2 shows an overview of our evaluation results. A feature is either present (marked as a green "Y"), not present (marked as a red "N"), or only supported partially, with manual coding, or through the use of extensions (marked as yellow "M"). Normally, such a comparison is made using scores and weights, as in [45]. But as scoring is highly dependent on the needs of the end-user, we omit this part.

Vendor Concerning the vendor pedigree, MS4 Me is the clear winner. RTSync is a spin-off of ACIMS, the creators of DEVS-Suite. ACIMS is headed by Dr. Bernard Zeigler, the founder of DEVS. RTSync, however, is still relatively young, and MS4 Me is still in beta. Other tools are developed by different research groups, specialized in DEVS simulation. Despite them being stable and usable, they are to be considered prototypes.

Documentation and sample models are provided by all tools. DEVS-Suite has the least documentation of all, as there is no user's guide beyond installation and running of the tool. PowerDEVS has limited documentation too: the user's guide link in the help menu doesn't respond, and the online manual is only partially filled in. However, the tool and its interface is fairly straightforward, even without documentation. Note that some parts of the $\mathrm{CD}++$ documentation require registration at their website.

For support, MS4 Me is again the clear winner, as it is the only one offering tuition and consulting services. On a commercial level, this is a crucial advantage.

Model and Input $\mathrm{CD}++$ and MS4 Me are the only tools providing an online library of models. But the size and reusability of these models is a different matter. $\mathrm{CD}++$ offers a sizable repository of models, containing a mix of reusable and stand-alone models. MS4 Me offers a relatively small model store, mostly containing example models instead of reusable models. PowerDEVS comes bundled with a small (offline) library too, though most models are oriented towards hybrid systems.

All tools involve coding to a certain degree. CD++, PowerDEVS, and VLE require coding at the level of atomic models, while offering a user-friendly format for the construction of coupled models. MS4 Me goes one step further, and presents a custom language for atomic models too. It is possible to manually write Java code, which will be included in the generated code. This allows the combination of the best of both worlds: basic conditionals and states using DNL, but data structure manipulations using Java.

Prepared using sagej.cls 


\begin{tabular}{|c|c|c|c|c|c|c|c|c|c|}
\hline & & $\begin{array}{l}\sum^{2} \\
\frac{1}{4} \\
\frac{1}{1}\end{array}$ & $\begin{array}{l}+ \\
\stackrel{+}{0}\end{array}$ & 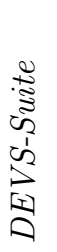 & \begin{tabular}{l}
$\sum_{\Sigma}^{0}$ \\
जै \\
\multirow{Z}{*}{}
\end{tabular} & 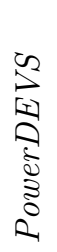 & 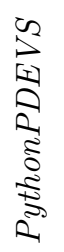 & $\stackrel{51}{5}$ & 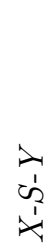 \\
\hline \multirow{3}{*}{ Vendor } & Pedigree & $\mathrm{N}$ & $\mathrm{N}$ & $\mathrm{N}$ & $\mathrm{Y}$ & $\mathrm{N}$ & $\mathrm{N}$ & $\mathrm{N}$ & $\mathrm{N}$ \\
\hline & Documentation & $\mathrm{Y}$ & $\mathrm{Y}$ & $\mathrm{N}$ & $\mathrm{Y}$ & M & $\mathrm{Y}$ & $\mathrm{Y}$ & $\mathrm{Y}$ \\
\hline & Support & $\mathrm{N}$ & $\mathrm{N}$ & $\mathrm{N}$ & $\mathrm{Y}$ & $\mathrm{N}$ & $\mathrm{N}$ & $\mathrm{N}$ & $\mathrm{N}$ \\
\hline \multirow{3}{*}{$\begin{array}{l}\text { Model } \\
\text { and input }\end{array}$} & Library & $\mathrm{N}$ & $\mathrm{Y}$ & $\mathrm{N}$ & $\mathrm{Y}$ & $\mathrm{Y}$ & $\mathrm{N}$ & $\mathrm{N}$ & $\mathrm{N}$ \\
\hline & Coding & $\mathrm{Y}$ & M & $\mathrm{Y}$ & M & M & $\mathrm{Y}$ & M & $\mathrm{Y}$ \\
\hline & Input & M & $\mathrm{Y}$ & $\mathrm{Y}$ & $\mathrm{Y}$ & M & M & $\mathrm{N}$ & M \\
\hline \multirow[t]{7}{*}{ Execution } & Speed control & $\mathrm{N}$ & $\mathrm{M}$ & $\mathrm{Y}$ & $\mathrm{Y}$ & $\mathrm{Y}$ & $\mathrm{Y}$ & $\mathrm{N}$ & $\mathrm{Y}$ \\
\hline & Multiple runs & $\mathrm{Y}$ & $\mathrm{Y}$ & $\mathrm{N}$ & $\mathrm{N}$ & $\mathrm{Y}$ & $\mathrm{Y}$ & $\mathrm{Y}$ & Y \\
\hline & Batch runs & $\mathrm{Y}$ & $\mathrm{Y}$ & $\mathrm{N}$ & $\mathrm{N}$ & $\mathrm{Y}$ & $\mathrm{Y}$ & $\mathrm{Y}$ & $\mathrm{N}$ \\
\hline & Parallel & $\mathrm{Y}$ & $\mathrm{Y}$ & $\mathrm{N}$ & $\mathrm{N}$ & $\mathrm{N}$ & $\mathrm{Y}$ & $\mathrm{Y}$ & $\mathrm{N}$ \\
\hline & Distributed & $\mathrm{N}$ & $\mathrm{Y}$ & $\mathrm{N}$ & $\mathrm{N}$ & $\mathrm{N}$ & $\mathrm{Y}$ & $\mathrm{Y}$ & $\mathrm{N}$ \\
\hline & Executable models & $\mathrm{Y}$ & $\mathrm{N}$ & $\mathrm{N}$ & $\mathrm{N}$ & $\mathrm{Y}$ & $\mathrm{N}$ & $\mathrm{N}$ & $\mathrm{N}$ \\
\hline & Termination condition & $\mathrm{Y}$ & $\mathrm{N}$ & $\mathrm{N}$ & $\mathrm{N}$ & $\mathrm{N}$ & $\mathrm{Y}$ & $\mathrm{N}$ & $\mathrm{N}$ \\
\hline \multirow[t]{5}{*}{ Animation } & Time Next & $\mathrm{N}$ & $\mathrm{N}$ & $\mathrm{Y}$ & $\mathrm{Y}$ & $\mathrm{N}$ & $\mathrm{M}$ & $\mathrm{N}$ & $\mathrm{N}$ \\
\hline & State & $\mathrm{N}$ & $\mathrm{Y}$ & $\mathrm{Y}$ & $\mathrm{Y}$ & $\mathrm{N}$ & M & $\mathrm{N}$ & $\mathrm{N}$ \\
\hline & Messages & $\mathrm{N}$ & $\mathrm{N}$ & $\mathrm{Y}$ & $\mathrm{Y}$ & $\mathrm{N}$ & M & $\mathrm{N}$ & $\mathrm{N}$ \\
\hline & Transitioning & $\mathrm{N}$ & $\mathrm{N}$ & $\mathrm{N}$ & $\mathrm{Y}$ & $\mathrm{N}$ & M & $\mathrm{N}$ & $\mathrm{N}$ \\
\hline & Sequence & $\mathrm{N}$ & $\mathrm{N}$ & $\mathrm{N}$ & $\mathrm{Y}$ & $\mathrm{N}$ & $\mathrm{N}$ & $\mathrm{N}$ & $\mathrm{N}$ \\
\hline \multirow{7}{*}{$\begin{array}{l}\text { Testing } \\
\text { and } \\
\text { Efficiency }\end{array}$} & Tracing & $\mathrm{Y}$ & $\mathrm{Y}$ & $\mathrm{Y}$ & $\mathrm{Y}$ & Y & $\mathrm{Y}$ & $\mathrm{Y}$ & Y \\
\hline & Step function & $\mathrm{Y}$ & $\mathrm{N}$ & $\mathrm{Y}$ & $\mathrm{Y}$ & Y & M & $\mathrm{N}$ & $\mathrm{Y}$ \\
\hline & Verification & $\mathrm{N}$ & $\mathrm{N}$ & $\mathrm{N}$ & $\mathrm{N}$ & $\mathrm{N}$ & $\mathrm{N}$ & $\mathrm{N}$ & Y \\
\hline & Backward clock & $\mathrm{N}$ & $\mathrm{N}$ & $\mathrm{N}$ & $\mathrm{N}$ & $\mathrm{N}$ & M & $\mathrm{N}$ & $\mathrm{N}$ \\
\hline & Interaction & $\mathrm{Y}$ & $\mathrm{Y}$ & $\mathrm{Y}$ & $\mathrm{Y}$ & M & $\mathrm{Y}$ & $\mathrm{N}$ & Y \\
\hline & Multitasking & $\mathrm{Y}$ & $\mathrm{Y}$ & $\mathrm{Y}$ & $\mathrm{Y}$ & $\mathrm{Y}$ & $\mathrm{Y}$ & $\mathrm{Y}$ & Y \\
\hline & Breakpoints & $\mathrm{N}$ & $\mathrm{N}$ & $\mathrm{N}$ & $\mathrm{N}$ & $\mathrm{N}$ & M & $\mathrm{N}$ & $\mathrm{N}$ \\
\hline \multirow[t]{2}{*}{ Output } & Delivery & $\mathrm{Y}$ & $\mathrm{Y}$ & Y & $\mathrm{Y}$ & $\mathrm{Y}$ & $\mathrm{Y}$ & $\mathrm{Y}$ & Y \\
\hline & Graphics & $\mathrm{N}$ & $\mathrm{Y}$ & $\mathrm{Y}$ & $\mathrm{Y}$ & M & $\mathrm{N}$ & $\mathrm{N}$ & $\mathrm{N}$ \\
\hline \multirow[t]{2}{*}{ User } & Orientation & $\mathrm{N}$ & $\mathrm{M}$ & $\mathrm{N}$ & $\mathrm{M}$ & $\mathrm{M}$ & $\mathrm{N}$ & $\mathrm{N}$ & $\mathrm{N}$ \\
\hline & Financial & $\mathrm{Y}$ & $\mathrm{Y}$ & $\mathrm{Y}$ & $\mathrm{N}$ & $\mathrm{Y}$ & $\mathrm{Y}$ & Y & $\mathrm{Y}$ \\
\hline
\end{tabular}

Table 2. General evaluation, based on $[8,45]$ 
Most tools allow user input during simulation, though differences exist in the kind of input. ADEVS requires the user to manually implement this functionality in the model and experiment. $\mathrm{CD}++$ reads input from a file, which is injected into the simulation at the desired time. DEVS-Suite and MS4 Me support graphical injection of events during simulation. PowerDEVS can take user input through the use of an input port. PythonPDEVS do not support user input, except during realtime simulation*. The PythonPDEVS debugging front-end [41], adds this feature for all kinds of simulation. VLE does not support input at all. X-S-Y reads its input from standard input, so the user either has to manually input the events, or write a wrapper script.

Execution Some tools include speed control, which is the option to run simulation using either scaled wall clock time (realtime simulation), or analytical time (as-fast-as-possible simulation). ADEVS and VLE are the only tools that don't support speed control by default. For CD++, the extended RT-CD++ [47] is required for realtime simulation.

Multiple runs, each with different parameters, are widely supported, but might require some coding from the user (i.e., writing the loop and the parameter variation). DEVS-Suite and MS4 Me don't support this at all, as these are GUI-only tools, and their GUI doesn't offer this function.

Batch runs are similar, except for X-S-Y, which waits for user input before terminating the simulation.

Parallel simulation is supported by ADEVS, CD++, PythonPDEVS and VLE. ADEVS allows this through the use of conservative synchronization, where each core is at a different point in simulated time. CD++, PythonPDEVS, and VLE offer distributed simulation, which can also be used for parallel simulation by hosting multiple nodes at the same machine. This generally imposes additional overhead due to the use of more general algorithms.

Many differences exist in terms of distributed simulation. $\mathrm{CD}++$, and in particular $\mathrm{PCD}++$, supports distributed simulation using different synchronization methods, provided by the Warped library [48]. PythonPDEVS uses Time Warp optimistic simulation, with MPI as the middleware. VLE does not support model distribution, but experiment distribution. A single model is not split up and distributed over multiple nodes, but the same model can be executed at different nodes simultaneously, using varying parameters. While this is fine when simulating multiple scenario's, a single model cannot be distributed or parallelized.

ADEVS and PowerDEVS support executable models, as they compile both the simulation tool and model into a single executable. All other simulators have models as files that are loaded by the simulator.

Termination conditions are included as an additional criteria. It determines how the modeller can specify when simulation terminates. Three different options were found: (1) step count, as found in DEVS-Suite and MS4

\footnotetext{
*In realtime simulation, simulation time is synchronized with the wallclock time, creating a linear relation between them [46].
}

Prepared using sagej.cls 
Me; (2) simulation time, as found in the others; and (3) termination condition, as found in ADEVS and PythonPDEVS.

With step counting, simulation halts after a predetermined number of simulation steps. Steps are unrelated to the simulation time, making it difficult for the user to predict exactly when simulation will terminate. The use of simulation time is more common, where simulation will halt at a predetermined point in the simulation. It has the advantage that it is closer to the problem domain. Should the model change (e.g., different parameters), simulation will still run up to the same point in simulated time. A termination condition is even closer to the problem domain, and allows the user to specify a termination state. As soon as this state is reached, simulation will terminate. This can be useful in design space exploration, where specific states in simulation are known to be unacceptable. Thus it becomes possible to quickly prune these branches, even before the usual termination time is reached.

Animation For animation, we deviate from the criteria due to these criteria not being distinguishing for our tools under study. We specify which part of the simulation algorithm is visualized. Message sequence visualization, to allow for manual dependency analysis, is another of our criteria. PythonPDEVS does not support any of these features, though its debugging extension does. As it is an extension, these entries are marked with an "M" even if they are fully present. The features that we selected for visualisation correspond to the different stages seen in the abstract simulator.

The first step of DEVS simulation is finding out which models are triggered at what time. DEVS-Suite and MS4 Me offer this with their simulation viewer: each model is anotated with the time remaining until internal transition, or the absolute time of its next transition.

The second step is state visualisation. Again, DEVS-Suite and MS4 Me support this by showing the name of the current state. CD++ can output a grid of floating point numbers, indicating the state of the cells in Cell DEVS simulation. After simulation, these values can be visualized with the "drawlog" tool that is provided with $\mathrm{CD}++$.

The third step is message visualisation. DEVS-Suite allows the visualization of the type of message exchanged (e.g., "Car"), but does not show the content. MS4 Me additionally visualizes event attributes (e.g., current velocity, maximal speed, or color).

The final step is transitioning model visualisation, optionally distinguishing between internal, external, and confluent transitions. DEVS-Suite does not support this, though it can be infered by manually following the exchanged messages. MS4 Me visualizes models that perform their transition by highlighting them, but makes no distinction between transition types. In PythonPDEVS, models executing their internal, external, or confluent transition are highlighted in distinct colors.

Additionally, MS4 Me allows simulation visualization with a sequence diagram. This sequence diagram visualizes exchanged messages, allowing for simple causality analysis.

Prepared using sagej.cls 
Testing and Efficiency All tools support tracing, though there is a distinction between textual, graphical, or both. Most textual output can be parsed, to allow for visualization with a different tool.

Simulation stepping is possible with several tools. ADEVS supports it, but the user must manually implement it. DEVS-Suite and MS4 Me offer support stepping through the simulation, combined with visualization. They also support stepping for a certain number of steps with a specified realtime scale. PowerDEVS only supports to execute a model for a specific number of steps. For PythonPDEVS, the debugging front-end can be used for stepping, as well as for visualization. X-S-Y allows stepping through its command line interface.

Verification is only supported by X-S-Y, but only for FD-DEVS models.

Backward clock, or stepping back, is only supported by the PythonPDEVS debugging extension.

All tools, apart from VLE, support simulation interaction. Different tools support different degrees of interaction: from high-level simulation control (e.g., pausing and resuming), to low-level state modification (e.g., the modification of model states at simulation-time).

Multitasking is supported by all tools, as they operate on text files instead of binary files. Multiple instances of a tool can be executed, allowing for multiple concurrent simulation executions. Models can be altered during simulation, using a text editor.

Breakpoints are only supported by the PythonPDEVS debugging extension.

Output All tools deliver some output, though often textual. Of special interest are $\mathrm{CD}++$, DEVS-Suite, and MS4 Me, which can create graphics of the simulation. PowerDEVS can make graphical output too, though this requires the use of built-in blocks from the library. Other tools require additional software to create graphical output.

For $\mathrm{CD}++$, these graphics are heatmap-like figures, which show the simulation state. For DEVS-Suite and MS4 Me, the graphics are simulation state traces, which show the simulation times at which some attribute of interest has changed.

User Due to the use of general-purpose programming languages, all tools are developer-oriented. An exception is MS4 Me, which uses a natural languagelike specification, combined with methods written in Java. This allows domain experts to work using DNL, while developers write utility functions in Java. $\mathrm{CD}++$ is also noteworthy, as Cell DEVS models can be completely specified using a custom language. With $\mathrm{CD}++$ Builder, it is also possible to use the DEVS-Graph formalism [2], which allows graphical construction of atomic DEVS models. A graphical front-end for PythonPDEVS also exists [49], which uses a neutral language. Although it still relies on writing code, the used language is specific to DEVS and can filter out illegal constructs, such as state modifications in the output function. All other tools require the modeller to write code in a general purpose programming language.

Prepared using sagej.cls 


\begin{tabular}{|c|c|c|c|c|c|c|c|c|c|}
\hline & & 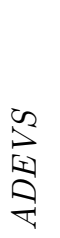 & $\begin{array}{l}+ \\
+ \\
0\end{array}$ & 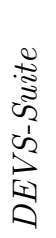 & $\begin{array}{l}\sum_{\Sigma}^{0} \\
\text { Wै } \\
\text { इ }\end{array}$ & 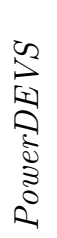 & 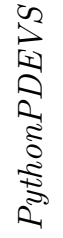 & $\stackrel{\sqrt[1]{3}}{=}$ & $\begin{array}{l}\lambda \\
=\dot{1} \\
\dot{y} \\
\dot{x}\end{array}$ \\
\hline \multirow[t]{4}{*}{ Formalisms } & Parallel DEVS & $\mathrm{Y}$ & $\mathrm{M}$ & $\mathrm{Y}$ & $\mathrm{Y}$ & $\mathrm{N}$ & $\mathrm{Y}$ & $\mathrm{Y}$ & $\mathrm{N}$ \\
\hline & Classic DEVS & $\mathrm{N}$ & $\mathrm{Y}$ & $\mathrm{N}$ & $\mathrm{N}$ & $\mathrm{Y}$ & $\mathrm{Y}$ & $\mathrm{N}$ & $\mathrm{Y}$ \\
\hline & Dynamic Structure & $\mathrm{Y}$ & M & $\mathrm{N}$ & $\mathrm{N}$ & $\mathrm{N}$ & $\mathrm{Y}$ & $\mathrm{Y}$ & $\mathrm{N}$ \\
\hline & Cell DEVS & $\mathrm{N}$ & $\mathrm{Y}$ & $\mathrm{N}$ & $\mathrm{N}$ & $\mathrm{N}$ & $\mathrm{N}$ & Y & $\mathrm{N}$ \\
\hline \multirow[t]{5}{*}{ Compliance } & Translation functions & $\mathrm{M}$ & $\mathrm{N}$ & $\mathrm{N}$ & $\mathrm{N}$ & $\mathrm{N}$ & $\mathrm{Y}$ & $\mathrm{N}$ & $\mathrm{N}$ \\
\hline & Event modularity & $\mathrm{N}$ & M & $\mathrm{N}$ & $\mathrm{N}$ & $\mathrm{N}$ & M & $\mathrm{N}$ & $\mathrm{N}$ \\
\hline & Positive time & $\mathrm{Y}$ & $\mathrm{N}$ & $\mathrm{N}$ & $\mathrm{M}$ & $\mathrm{N}$ & $\mathrm{Y}$ & Y & $\mathrm{N}$ \\
\hline & Select function & - & $\mathrm{N}$ & - & - & M & $\mathrm{Y}$ & - & $\mathrm{N}$ \\
\hline & Confluent & $\mathrm{Y}$ & - & $\mathrm{Y}$ & $\mathrm{N}$ & - & $\mathrm{Y}$ & $\mathrm{Y}$ & - \\
\hline
\end{tabular}

Table 3. DEVS-specific evaluation, loosely based on [6]

Financially, all tools are open-source and freely available, except for MS4 Me, which is proprietary and requires a paid license. Installation and maintenance costs should also be considered, though these are difficult to estimate. Familiarity of the modellers with the used language is also of importance, as otherwise a significant amount of training is required.

\section{DEVS-specific evaluation criteria}

Some additional criteria were added to check for strict conformance to the DEVS formalism, based on a previously defined set of criteria [6]. Several were dropped, whereas some were added to extend the criteria to Parallel DEVS.

First, the supported formalisms of all tools are compared. Only the four previously introduced DEVS formalisms are considered here. Some tools support additional formalisms. An overview is shown in Table 3.

The remainder of this paper will not present examples and performance results of either Cell-DEVS or Dynamic Structure DEVS, as these are not widely supported by the tools under study. Similarly, we do not go deeper into some other aspects of DEVS simulation, such as hybrid simulation and HLAcompliance: most tools under study have only limited (if at all) support for both. A comparison detailing any of these aspects, either through detailed features or performance analysis, would require a different set of tools under study to make a fair comparison.

Supported formalisms Each simulation tool sets out to support a different set of formalisms. Parallel DEVS, the successor of Classic DEVS, is supported in all tools, except for $\mathrm{CD}++$, PowerDEVS, and $\mathrm{X}-\mathrm{S}-\mathrm{Y}$. For CD++, this is because we used N-CD++ instead of PCD ++, which is a Classic DEVS simulator instead of a Parallel DEVS simulator. For X-S-Y and PowerDEVS, no Parallel DEVS version is available at the moment.

Prepared using sagej.cls 
Classic DEVS is used in those tools that don't support Parallel DEVS. PythonPDEVS supports both Parallel DEVS and Classic DEVS. The former for performance, and the latter for support for legacy models.

On top of the previously defined DEVS formalisms, some tools offer dynamic structure. Due to the variety of dynamic structure formalisms, such as DSDEVS [12] and DynDEVS [17], we have grouped all of these under a common term. A modified version of $\mathrm{CD}++[28]$ exists, which supports dynamic structure.

Finally, CD ++ and VLE have specific modelling and simulation options for Cell DEVS models.

DEVS compliance Our first criteria is the presence of the translation function, which is the function denoted by the $Z_{i, j}$ in the formal definition. It translates event from output-to-input, output-to-output, and input-to-input ports. Despite the arguments in favor of this function, only PythonPDEVS implements this function. For ADEVS, it is possible to overload the routing mechanism, introducing message modification there.

DEVS is a modular formalism: models can only communicate through event exchange. There is no way to read or change the state of another model, except through the exchange of an event which causes the model to alter its own state. While breaking modularity allows for improved performance [50], modularity is a necessity in DEVS as it is the basis of its closure under coupling. Due to the use of general-purpose programming languages in the models, several ways to break modularity exist. While some limitations can be imposed (e.g., preventing the passing of pointers or references in events), there is always the possibility to abuse language constructs (e.g., global variables). PythonPDEVS partially enforces modularity by making deep copies of exchanged events. CD ++ also enforces modularity in this respect. Though, this is mainly caused by restriction of events, which can only be floating point values. Some work has been done on using static analysis of models, using a neutral language, to prevent such constructs [49].

A third requirement is for the Time Advance function to be nonnegative. As a negative time advance is clearly impossible in reality, this is disallowed in a DEVS model as well (though a time advance of 0 is allowed). Most tools do not check this, assuming that the user follows the formalism, and can therefore give incorrect simulation results. A notable case is MS4 Me: statically detectable negative time advances are flagged as modelling errors, though there is no run-time check for dynamically obtained values.

A fourth requirement is the presence of a select function, which only applies to Classic DEVS models. The select function determines the model to execute in case multiple models are scheduled to execute an internal transition function at the same time. Of the four tools supporting Classic DEVS, only PythonPDEVS allows users to define the select function explicitly. PowerDEVS allows the definition of a priority list, though it is not possible to define arbitrary functions. $\mathrm{CD}++$ and $\mathrm{X}-\mathrm{S}-\mathrm{Y}$ implicitly use a hard-coded select function, such as selecting the first model after alphabetic sorting on model name.

Prepared using sagej.cls 
Our final requirement is the presence of a confluent transition function, which only applies to Parallel DEVS models. The confluent transition function is triggered in case both the internal and external transition should fire at exactly the same point in time. All of the Parallel DEVS simulators support this requirement, except for MS4 Me, where a default is assumed. Other tools often provide the same default, though the user can override this default.

\section{Performance}

With the growing demand for computing resources by modern simulation applications, the need for efficient simulators increases. Parallel and Distributed Simulation (PaDS) thus becomes necessary to allow multiple computers to cooperatively work on a single simulation. Distribution and parallelism do not solve all problems though, as some problems are inherently difficult to parallelize. Efficient sequential algorithms therefore stay relevant, as parallel and distributed synchronization algorithms are a layer on top of sequential algorithms.

As not all of our tools under study support parallel or distributed simulation - and even those that support it, use very different synchronization protocols, making a fair comparison difficult — we have opted to only study sequential performance.

All simulations were performed on a system with an Intel i5-4570 (3.2 GHz) with 16GB of DDR3-1600 main memory, running Gentoo Linux with kernel version 3.18.22. We used the following software versions: GCC 4.9.3, OpenJDK IcedTea6 1.13.9, Python 2.7.10, and PyPy 2.6.0. All tools were compiled with the optimizations defined in their Makefile. For ADEVS, we had to make a Makefile ourself, where we opted for the compiler flag "-O2". MS4 Me was benchmarked on the same machine, but using Windows 7 Enterprise SP1.

All of the source code used for this paper (i.e., all models, benchmarks, and a copy of the tools used where possible) can be found at http://msdl.cs . mcgill.ca/people/yentl/DEVS/tools.tgz.

Tools written in Python were benchmarked using both CPython ${ }^{\dagger}$ (the reference implementation) and $\mathrm{PyPy}^{\ddagger}$ (an alternative implementation using JIT compilation). As most of the inefficiency of Python code is caused by its interpreted nature, we can partially mitigate this with $\mathrm{PyPy}$, which uses justin-time compilation. While PyPy can be used in cases where performance is important, most users will prefer to use CPython as it is installed by default on most Linux distributions. We have opted to include both: CPython for the average user, who is unconcerned about performance, but also $\mathrm{PyPy}$, for power users. As PyPy is (almost) a drop-in replacement for CPython, it is possible to develop and prototype using CPython, but perform the actual long-running simulations using PyPy.

${ }^{\dagger}$ https://www.python.org/

${ }^{\ddagger}$ http://pypy.org/

Prepared using sagej.cls 


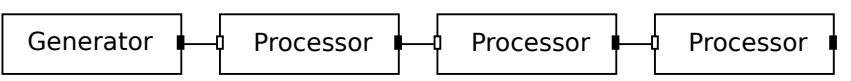

Figure 7. "Queue" model: every output port is connected to the input port of the previous model. Shown for 3 models (excluding the generator).

We present the benchmark, their results, and discuss the results.

\section{Benchmarks}

Three different benchmark models are used, offering insight in the performance of different aspects of the simulation algorithms. Of these three, two are synthetic, and one is more realistic, though still fairly simple.

For the synthetic benchmarks, we looked into the DEVStone [9] benchmark for inspiration. DEVStone defines 4 kinds of models: (1) LI: models without a lot of interconnection, (2) HI: models with a lot of interconnection, (3) HO: HI models with lots of outputs, (4) HOmod: models with an exponential level of coupling and outputs. The transition functions of each model contains artificial computation, in the form of Dhrystones.

We found some limitations to the default DEVStone models, such as all atomic models triggering their external (and possibly internal) transition simultaneously, which is unlikely in realistic DEVS models. The execution of Dhrystones during the transition functions doesn't fit our desired analysis either: some of our tools are written in different programming languages, each with distinct performance characteristics. As the operations in the transition functions are time-bounded, a more complex (i.e., more computation) model is executed in efficient programming languages. For a fair comparison, we would like all tools to simulate an identical model, that is, without any computation in the transition functions. Overhead of the simulation is no longer computable, as there is no longer any "theoretical simulation time", as was the case in the original definition [9]. We can, however, compare total execution times for a specific model in a specific configuration. By minimizing the amount of model computation, execution times maximally show the time taken by the simulation kernel.

The use of deep hierarchy is not considered in our benchmarks, as models that are hundreds of levels deep are unrealistic. Additionally, modern simulation tools often provide automatic flattening, creating a single coupled model with all atomic models as its direct children. This situation closely mimics the models of our proposed benchmarks. Note that, whereas flattening as a positive effect on most realistic models, it is not necessarily always an optimization: depending on model structure, the flattening overhead might be significant compared to the small gain.

There is no doubt that the benchmark implementations, for all simulation tools, can be improved for both code efficiency, simulation performance, and conciseness.

Prepared using sagej.cls 


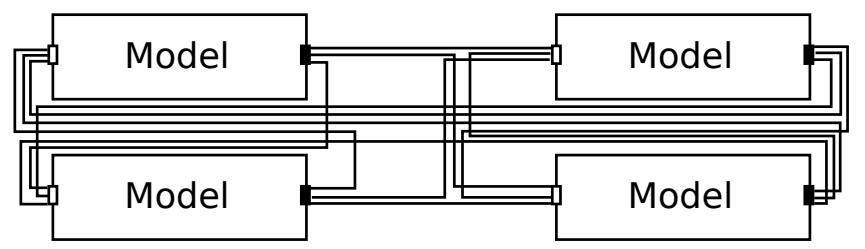

Figure 8. "High Interconnect" model: every output port is connected to every input port of a different model. Shown for 4 models.

Queue model The "Queue" is a simple model, where a single generator periodically creates output and sends it to the first processor. Each processor sends the output to exactly one other processor, called its successor. There are no loops in the connections, thus forming a single line of processors, as shown in Figure 7. If a processor receives a new event while processing an event, the event is placed in a FIFO queue. After processing an event, the processor will check its queue and pop an event to process.

Our Queue model bears similarity to the HI DEVStone model, in the sense that models are connected to their successors, but also to the LI DEVStone model, as model output is only connected to a single other model. We did not completely take over the HI model, as otherwise all models would receive input at the exact same time, thus triggering all external transitions (and later on, their internal transitions) simultaneously. This is an unnatural occurence in DEVS, which specifically uses a continuous time-base. On the other hand, a benchmark model with a low number of inter-model connections is necessary. The Queue model is the result of this merge.

It is still interesting to analyse the behaviour of the simulation kernel when transition functions are triggered simultaneously (called collisions from now on). We allow for either a "full collision" model or a "no collision" model, by defining the time advance function as either fixed (to 1) or random (uniformly distributed between 0 and 2), respectively. If the time advance function always returns a fixed number, all models will transition at exactly the same time. Otherwise, the time advance function returns a random number, preventing most collisions.

High Interconnect model The "High Interconnect" model is similar to the queue, but has a lot of connections. Every model is connected to every other model, as shown in Figure 8. Each model outputs an event, which is routed to all other models. Upon the reception of an event, the models trigger their external transition function, which simply ignores the message.

This model is a more complex form of the HI DEVStone model, where instead of only 1 outgoing connection, multiple outgoing (and incoming) connections are made. The number of connections, and consequently of exchanged events, scales quadratically.

This not only benchmarks the performance in the presence of a high number of connections, but also of routing a single event to a multitude of receivers. A parameter is again provided to define whether or not collisions should happen.

Prepared using sagej.cls 


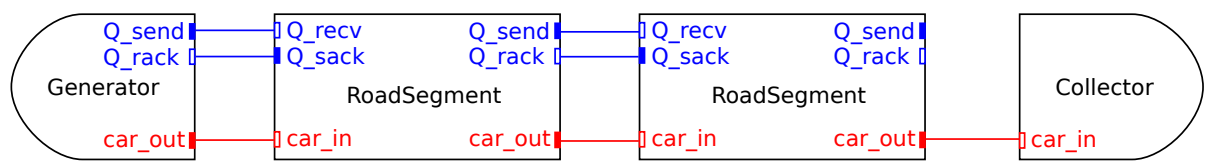

Figure 9. "Traffic" model, shown for 2 segments.

In case collisions happen, the bag merging algorithms of Parallel DEVS are benchmarked too, as every model outputs an event to every other model. All events then need to be merged into a single bag.

Despite that this model might seem totally unrealistic, it was added to monitor the efficiency of simulation algorithms in case many events were exchanged and processed simultaneously. Whereas other, more realistic, benchmarks could be conceived to monitor this, they would go further away from the core aspects that are to be monitored: event exchange. By only keeping the actual aspects to be monitored, performance results are less cluttered with other aspects of the model. Additionally, while such models might be ill-suited for DEVS, sometimes DEVS is used for compatibility reasons, as argued in [1]. Whether or not DEVS is the ideal formalism for this part of the model is then less relevant: it needs to be used to interact with other models, and even then, high performance is very relevant.

Traffic model The "Traffic" model is a more realistic model [51]. It resembles the Queue model without collisions, though more realistic communication and computation patterns occur.

It consists of a generator, some road segments, and a collector, as shown in Figure 9. After a randomly sampled time, a car is generated by the generator. The generator outputs the car and sends it to the connected road segment. Every road segment processes the car for a certain time (depending on the velocity), after which it is sent to the next road segment. A car can accelerate or decelerate, depending on their prefered speed, the speed limit of the road segment, and the cars in front of them. To prevent car collisions, road segments communicate with each other through the use of queries and acknowledgements. As soon as a road segment receives a new car, it sends a query to the next road segment, requesting whether the next road segment is free. It gets an acknowledgement back, stating how long it will take for the road segment to become available. The car at the current road segment will adjust its speed accordingly, depending on the maximal acceleration and deceleration values. If a road segment does not receive an acknowledgement in time, the car goes on to the next road segment without adjusting its speed. At the end of the road segments, a collector receives all cars and computes average velocity and average deviation from the prefered velocity. These statistics are used to test the correct implementation of the model in the various simulation tools.

Prepared using sagej.cls 


\begin{tabular}{|c|c|c|c|c|c|c|c|c|}
\hline & 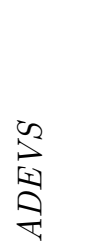 & 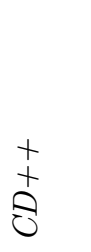 & 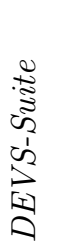 & $\begin{array}{l}\stackrel{\Sigma}{\Sigma} \\
\text { जै } \\
\sum_{z}^{*}\end{array}$ & 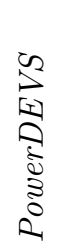 & 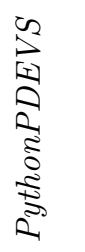 & $\begin{array}{l}\sqrt[1]{3} \\
\leq\end{array}$ & $\begin{array}{l}\lambda \\
w^{\prime} \\
\dot{1} \\
\dot{x}\end{array}$ \\
\hline Queue & 178 & $169^{*}$ & 152 & $113^{*}$ & 234 & 94 & $150^{*}$ & 104 \\
\hline Highly connected & 112 & $103^{*}$ & 113 & $57^{*}$ & 182 & 64 & $73^{*}$ & 76 \\
\hline Traffic & 609 & $672^{*}$ & 498 & $380^{*}$ & 473 & 345 & $599^{*}$ & 359 \\
\hline
\end{tabular}

Table 4. Lines of code for the benchmarks. ${ }^{*}$ indicates that this is excluding the experiment file and coupled models.

\section{Source code size}

For every tool and every benchmark, Table 4 shows the lines of code used to implement the model. This number includes the atomic models, coupled models, and experiment file. Entries marked with a ${ }^{*}$ only count the size of the atomic models, as other parts are constructed graphically (VLE), or using a verbose syntax $(\mathrm{CD}++, \mathrm{MS} 4 \mathrm{Me})$. DEVS-Suite models do not contain code for experiment setup, as this is done manually by the user.

This analysis does not go much further than the differences already known [52]: the same behaviour in $\mathrm{C}++$ requires more lines of code than in Java, which still requires more than in Python. Recall that MS4 Me uses a natural language-like language. This has a significant impact on the total size of the models, though it seems that Python still requires less lines of code. In essence, the natural language is very concise, though the total number of lines of code is still this high due to the use of some Java code in the model. As such, it is more appropriate to compare MS4 Me to DEVS-Suite, as DEVS-Suite models are written completely in Java.

PowerDEVS has the highest number of lines of code, which is due to the (helpful) comments that are included by default in all models. As these benchmark models are relatively small, their overhead becomes significant. For the traffic benchmark though, the overhead is negligible, resulting in a smaller codebase. $\mathrm{CD}++$ has the most verbose code for the Traffic model, since complex events are expanded: all event attributes are passed seperately over their own ports, resulting in more verbose code.

A complete usability study is outside of the scope of this paper, so we limit ourself to the size of the model. As most tools resort to programming for atomic models, it is best to chose a simulation environment that uses a language which the modeller is familiar with. If the modeler is unfamiliar with any programming language, MS4 Me provides the simplest language to express model behaviour.

Prepared using sagej.cls 


\section{Remarks}

Due to the comparison of different tools, each with their own design decisions (e.g., about implementation language and required input format), some remarks are required on how these results should be interpreted.

First, different representations are used for coupled models. Tools such as ADEVS use programming language constructs to create a set of models and couple them. This is highly efficient and allows modellers, familiar with programming techniques, to create arbitrary constructions. Tools such as VLE use a graphical environment to create the models. Coupled models are no longer represented with programming language constructs, but using a custom syntax. This custom syntax, like XML in VLE, induces additional overhead during simulation, as it needs to be parsed. The overhead is only required during initialization, though it imposes an overhead compared to the other tools. Our results include this initialization overhead, as it is required at every simulation run. Tools using compiled models do not have their compilation time included, as compilation is only required once. Compilation times are negligible in long-running simulations, or when a single (compiled) model is used in various settings. During prototyping, compilation times of several seconds, as is the case with ADEVS, offset the higher simulation performance. Some tools also perform some preprocessing of the model, such as direct connection [53]. Whether or not the compilation and initialization overhead is tolerable, compared to the simulation time, is a decision that has to be left to the end-user.

Second, DEVS-Suite and MS4 Me do not provide a command line interface, so we are unable to benchmark them automatically. Results for these simulators were obtained by manually starting and stopping the simulation, while measuring the time. As a result, these measurements have a lower accuracy and precision. Being purely graphical, they continously update their GUI with simulation information (e.g., current simulation time and number of processed transitions). We disabled as much of these visualizations as possible to obtain our results, though a significant overhead is to be expected due to this animation.

Third, MS4 Me has low performance in all simulation benchmarks. This is possibly caused by the tool still being in beta status, meaning that some functionality is still missing or not completely implemented, or just that development effort is currently not focussed on performance optimization. For example, all transitions are logged to the console, inducing a significant overhead. A more significant problem is that simulation performance seems efficient at first, but quickly grinds to a halt. Our analysis shows that this is caused due to high memory usage, resulting in very frequent garbage collection, finally even causing out-of-memory errors. This could indicate the presence of a memory leak. Through some undocumented options ${ }^{\S}$ it was possible to avoid this out-of-memory error and significantly increase the performance. Nonetheless, the results that were included here are using this trick, but still performance

\footnotetext{
$\S$ Manually invoking the generated Java files instead of running them through the GUI.
} 
is significantly lower than other DEVS simulation tools. As a result of these problems, results for MS4 Me are likely to change significantly in the future.

Fourth, as there was a huge variety in simulation performance, we plotted all tools on a logarithmic scale, and the fastest few on a linear scale.

Fifth, results when using PyPy might seem strange in comparison to the other results. This is caused by the JIT, which requires some warm-up first. For small models, the total simulation time is dominated by the interpretation phase and the JIT compiler compiling the code. Therefore, these results are fairly inaccurate for short simulations.

Sixth, CD++, PowerDEVS, and X-S-Y are Classic DEVS simulation tools, whereas the others use Parallel DEVS. Despite identical model behaviour, both formalisms mandate different simulation algorithms, causing differences in simulation performance. We argue that they are still comparable, since they are equally expressive, and no simulation tool (except for PythonPDEVS) supports both at the same time. This means that users are stuck with the formalism of the tool they chose.

\section{Queue Results}

Results for the Queue benchmark are shown in Figure 10. The results indicate that ADEVS is fastest in both cases, with PowerDEVS coming extremely close.

For a random time advance, as in the left part of Figure 10, no collisions occur. For the fixed time advance, as in right part of Figure 10, collisions always occur. Several important differences can be seen when comparing the two.

First, DEVS-Suite is a lot faster with collisions than without collisions. There can be several reasons for this. One option is that their main simulation loop is inefficient (e.g., due to GUI updating), as far fewer simulation steps are executed if all models collide.

Second, PythonPDEVS outperforms VLE in the presence of collisions. This can be explained due to the activity-based data structures implemented in PythonPDEVS [54]. As a list-based scheduler would be better in case lots of collisions occur [22], the data structure modifies itself to a list-based one. VLE and ADEVS have a static, heap-based, scheduler, which is not optimized for this situation.

Third, using PyPy instead of CPython has a significant impact on both PythonPDEVS and X-S-Y. With CPython, X-S-Y is far slower than PythonPDEVS, though they come much closer when using PyPy. This indicates that much of the performance of PythonPDEVS with CPython is gained due to CPython-aware optimizations (e.g., expensive function calls), which are largely mitigated using PyPy (e.g., using JIT inlining).

Fourth, ADEVS and PowerDEVS come very close. This likely indicates that they implement similar algorithmic optimizations, and that the compiler optimizes out most implementation details.

Prepared using sagej.cls 

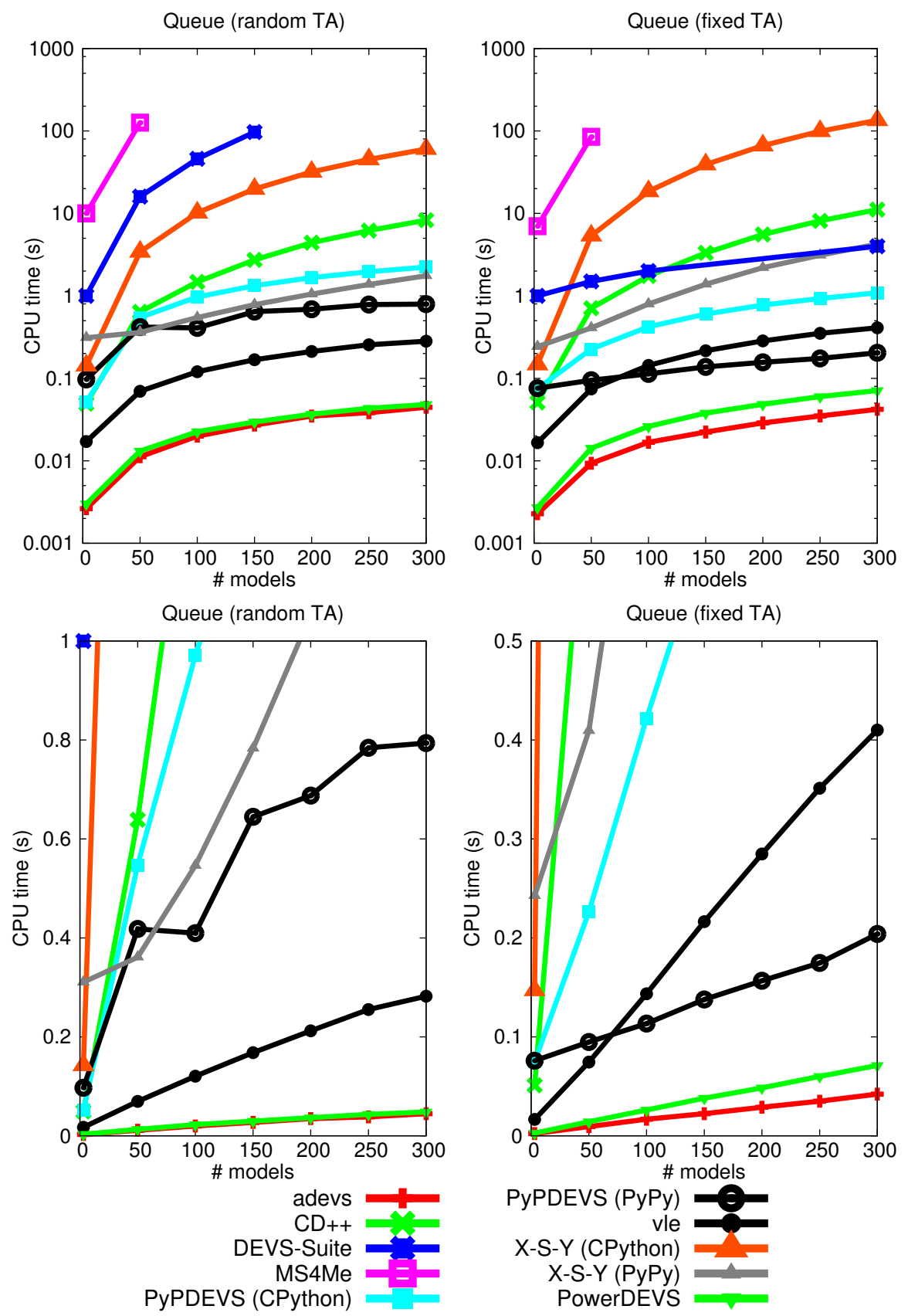

Figure 10. Benchmark results for the "Queue" benchmark. Top figures use a logarithmic scale, bottom figures are zoomed in on the fastest tools and uses a linear scale. 

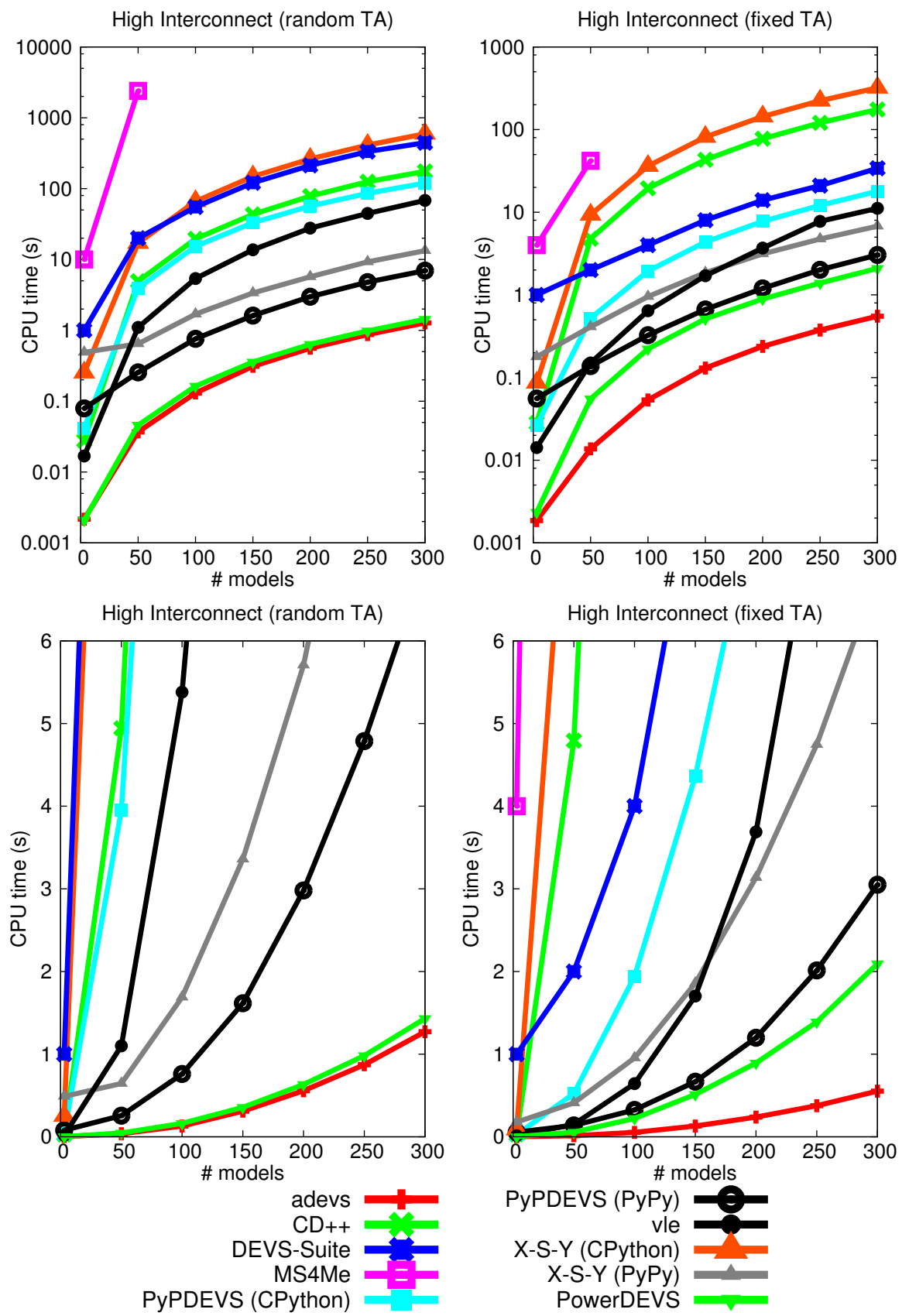

Figure 11. Benchmark results for the "High Interconnect" benchmark. Top figures use a logarithmic scale, bottom figures are zoomed in on the fastest tools and uses a linear scale. 


\section{High Interconnection Results}

Results for the High Interconnection benchmark are shown in Figure 11. Again, ADEVS is clearly the fastest, with PowerDEVS coming close in case of a random time advance.

For PowerDEVS, the reason for this slower performance mostly lies with the use of Classic DEVS, where other tools (except CD++ and X-S-Y) use Parallel DEVS. In the presence of simultaneous events, Classic DEVS models trigger their select function, selecting a single model. For $n$ colliding models, as is the case with a fixed time advance, this causes $n$ seperate lookups in the scheduling data structures, explaining the difference. This same occurence is aggrevated here, as every model needs to trigger its external transition once for every other model. Each external transition is called $n$ times more in Classic DEVS simulation kernels due to the formalism. As external transitions contain nearly no computation, this effect is not clearly visible. Because this was not the case in the previous benchmark, the difference between Classic DEVS and Parallel DEVS was relatively small.

VLE performs much slower than expected, even slower than X-S-Y (using PyPy), certainly in the absence of simultaneous events. With simultaneous events, the same behaviour can be seen, though VLE is now much closer to XS-Y, and PythonPDEVS further distinguishes itself from the others. We expect this low performance to be due to the high number of connections, which need to be parsed at simulation time.

DEVS-Suite again shows better results in the presence of simultaneous events, just like in the previous benchmark.

\section{Traffic Results}

Figure 12 shows the results of the Traffic benchmark. Results are very similar to those for the "Queue" model, as the basic principles are the same. Once again, ADEVS and PowerDEVS are tied, followed by VLE. Some differences are visible because the transition functions are now more complex, and more event passing happens.

\section{Conclusions on performance}

Our analysis has shown that ADEVS is currently the fastest for Parallel DEVS, and PowerDEVS for Classic DEVS. Their performance is unmatched in any kind of model we have tried, leaving competitors behind by a fair margin. However, this performance comes at the cost of functionality and debugability. Debugging ADEVS models, written in $\mathrm{C}++$, combined with ADEVS's meager debugging capabilities, is significantly more work than with other simulation tools. For example, there is no tracing functionality included by default. PowerDEVS includes a minimal simulation tool in the model, but at least contains a debugging feature which traces the methods being invoked.

VLE comes fairly close in more realistic models, but loses terrain when the model has many connections. While this is a fairly uncommon case, it

Prepared using sagej.cls 

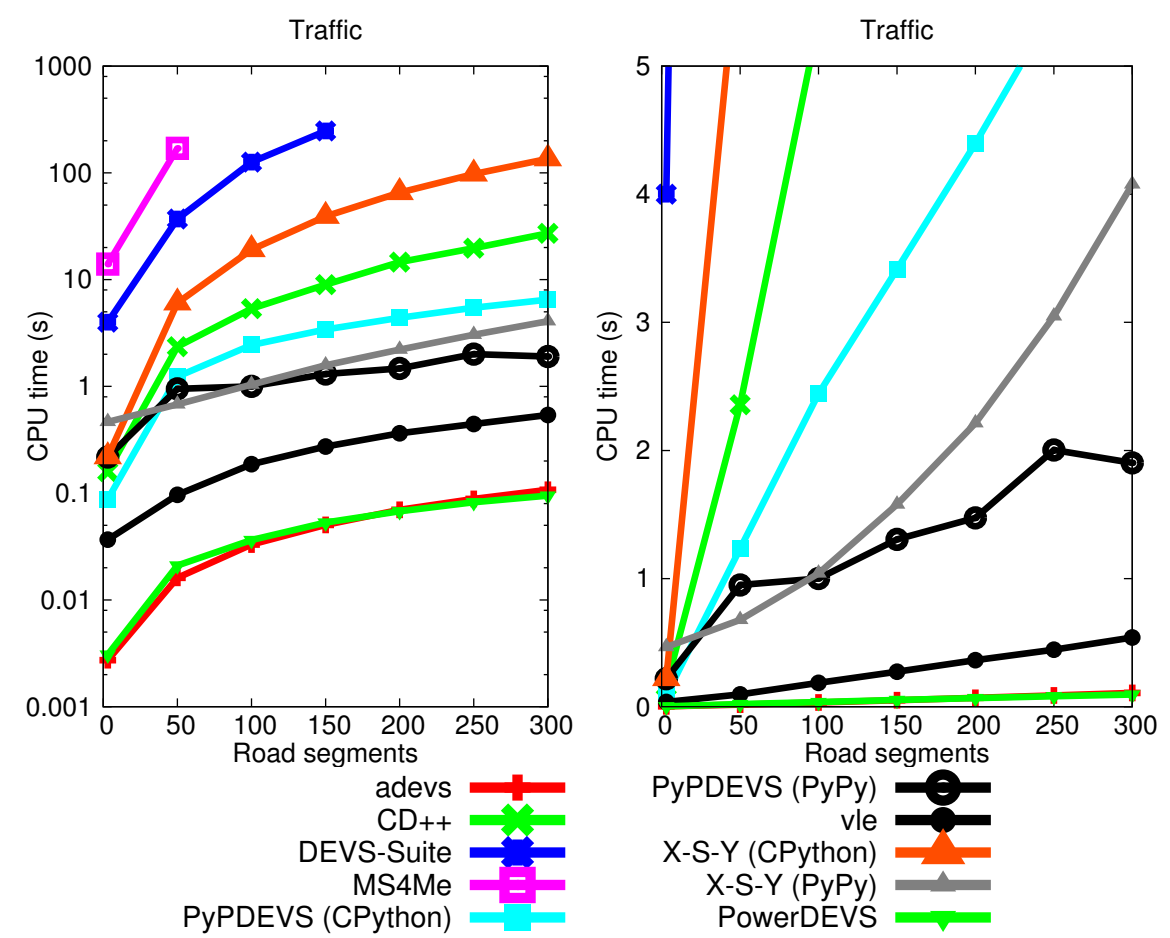

Figure 12. Benchmark results for the "Traffic" benchmark. The left figure uses a logarithmic scale, whereas the right figure is zoomed in on the fastest tools and uses a linear scale.

is remarkable that this simulation kernel falls that far behind in this specific configuration. The performance gap with ADEVS is most likely caused by the use of an XML-based language for coupled models, which needs to be parsed at simulation time.

PythonPDEVS comes in third for the Queue model, and clearly profits from its activity-based scheduler, as it comes quite close to VLE for models with many collisions. For models with a high number of connections, it comes in second due to the suddenly degraded performance of VLE. Combined with the use of an interpreted language, thus not needing compilation, PythonPDEVS is useful for prototyping, even of large scale models. Simulation using CPython is clearly slower than using PyPy, though even simulation using CPython is not amongst the slowest.

X-S-Y comes in fourth, though sometimes falls behind due to the use of Classic DEVS. The performance gap between CPython and $\mathrm{PyPy}$ is much bigger than it was for PythonPDEVS. PythonPDEVS has many optimizations built-in that avoid slow paths in CPython, reducing the potential speedup of PyPy. Such optimizations are still important, as many users will use CPython instead of PyPy. This is certainly true for users who don't seek maximal performance.

Prepared using sagej.cls 
$\mathrm{CD}++$ comes in fifth, even though it is implemented in $\mathrm{C}++$. A likely explanation is the use of a custom language for coupled models, which is parsed at simulation time.

DEVS-Suite seems to be inefficient in a few situations, though fairly efficient in some others. These results are likely related to the GUI, which was constantly updating the textual values, even with the graphical simulation view disabled. Other simulation tools do not provide the same level of detail on the running simulation, nor do they offer visualization of the running simulation.

Lastly, MS4 Me could only be used for relatively small models, due to low performance. Without fixing the memory leak issue, even these relatively small models were unable to be simulated until their termination time.

\section{Conclusion}

We have shown that many differences exist between DEVS simulators. Differences ranged not only between functionality and performance, but also between the programming languages used for modelling. The lack of a standardized DEVS representation, in a programming language-independent form, causes models to be incompatible between simulators. Due to the arbitrary cost of porting models between different simulation tools, and different programming languages, simulation tool lock-in can occur. This makes the initial choice of tooling an important decision. Our comparison aims to aid in this important decision.

The final decision on which is the "best" tool is dependent on the requirements set out by the team that will be using the tool. Performance analysis showed the expected results: low-level simulation tools achieve significantly higher performance, at the cost of reduced readability and (debugging) functionality. We briefly summarize each tool, and give a recommended target audience:

- ADEVS offers a limited set of features, but allows for very efficient simulation of Parallel DEVS models. It is however difficult to use by non-programmers as it boils down to programming in $\mathrm{C}++$. We would recommend the use of ADEVS for modellers that are familiar with $\mathrm{C}++$ and wish to obtain every bit of performance, at the cost of functionality.

- $C D++$ is mainly specialized in the simulation of Cell DEVS models by nonprogrammers. While Classic DEVS models are also supported, extensions such as $\mathrm{CD}++$ Builder are recommended for non-programmers. We would recommend the use of $\mathrm{CD}++$ for the development of Cell DEVS models, or in combination with $\mathrm{CD}++$ Builder.

- DEVS-Suite presents a nice simulation environment that provides much insight in the semantics of Parallel DEVS models. Because of its additional features, performance is rather slow, making it unsuited for large scale simulations. We would recommend the use of DEVS-Suite for educational purposes, where the steps of DEVS simulation needs to be thoroughly explained.

Prepared using sagej.cls 
- MS4 Me provides an intuitive modelling and simulation environment that tries to hide programming at the level of both atomic and coupled models. The availability of consulting options distinguishes it from the rest, though it is still in beta and performance is insufficient for any reasonably sized model. We would recommend the use of MS4 Me only as soon as the tool has stabilized in terms of performance, after which it is a tool appropriate for non-programmers.

- PowerDEVS offers an integrated modelling environment, though it still relies on the modeller writing $\mathrm{C}++$ code. Its performance is often on par with ADEVS, though it only supports Classic DEVS, despite Parallel DEVS being the more popular formalism nowadays. We would recommend the use of PowerDEVS for modellers familiar with $\mathrm{C}++$, but not so familiar with DEVS itself, or those seeking to use DEVS for the simulation of hybrid systems.

- PythonPDEVS provides users with a DEVS simulator in Python, offering features that are relevant to beginning users of DEVS. While performance is decent compared to most other tools, it is vastly outperformed by other efficient simulation tools. We would recommend the use of PythonPDEVS for educational purposes (due to its close compliance to the formalism), or for prototypes (due to its relatively efficient implementation in Python).

- $V L E$ provides an integrated modelling environment similar to PowerDEVS, but remains at a more basic level. There is, however, more support for the creation of experiments and execution on multiple machines. We would recommend the use of VLE when a single environment is desired for every operation: from writing utility functions in $\mathrm{C}++$ to running experiments with various configurations.

- $X-S-Y$ is unique in that it offers support for a verifiable subset of DEVS, implemented in Python. Performance is lacking though, making it unfit for large scale models. We would recommend the use of X-S-Y if model verification is important.

Some parts were left out of our comparison, such as an analysis of parallel and distributed simulation performance, or a more detailed performance comparison (e.g., memory usage). Usability evaluation of the tools is an important aspect that we did not tackle. Our intuition tells us that graphical tools can make up for their low performance, by significantly reducing required training and model development time. The same goes for debugability: tools offering advanced debugging capabilities are orders of magnitude slower, though model errors are likely found earlier. In the future, performance analysis of the parallel and distributed simulation should be considered, as well as usability evaluation of these tools.

Prepared using sagej.cls 


\section{Acknowledgement}

This work was partly funded with a $\mathrm{PhD}$ fellowship grant from the Research Foundation - Flanders (FWO). This research was partially supported by Flanders Make vzw.

\section{References}

[1] Vangheluwe H. DEVS as a common denominator for multi-formalism hybrid systems modelling. In IEEE International Symposium on ComputerAided Control System Design. pp. 129-134.

[2] Bonaventura M, Wainer G and Castro R. Graphical modeling and simulation of discrete-event systems with CD++Builder. SIMULATION 2013; 89(1): 4-27.

[3] Vicino D, Niyonkuru D, Wainer G et al. Sequential PDEVS Architecture. In Proceedings of the 2015 Spring Simulation Multiconference. pp. 906-913.

[4] Gutierrez-Alcaraz M and Wainer G. Experiences with the DEVStone Benchmark. In Proceedings of the 2008 Spring Simulation Multiconference. pp. $447-455$.

[5] Wainer G, Glinsky E and Gutierrez-Alcaraz M. Studying performance of DEVS modeling and simulation environments using the DEVStone benchmark. SIMULATION 2011; 87(7): 555-580.

[6] Li X, Vangheluwe H, Lei Y et al. A testing framework for DEVS formalism implementations. In Proceedings of the 2011 Spring Simulation Multiconference. pp. 183-188.

[7] Franceschini R, Bisgambiglia PA, Touraille L et al. A survey of modelling and simulation software frameworks using Discrete Event System Specification. In 2014 Imperial College Computing Student Workshop. Schloss Dagstuhl-Leibniz-Zentrum fuer Informatik, pp. 40-49.

[8] Nikoukaran J, Hlupic V and Paul RJ. Criteria for simulation software evaluation. In Proceedings of the 1998 Winter Simulation Multiconference. pp. 399-406.

[9] Glinsky E and Wainer G. DEVStone: a benchmarking technique for studying performance of DEVS modeling and simulation environments. In Proceedings of the 2005 9th IEEE/ACM International Symposium on Distributed Simulation and Real-Time Applications. pp. 265-272.

[10] Zeigler BP, Praehofer H and Kim TG. Theory of Modeling and Simulation. 2nd ed. Academic Press, 2000.

[11] Chow ACH and Zeigler BP. Parallel DEVS: a parallel, hierarchical, modular, modeling formalism. In Proceedings of the 1994 Winter Simulation Multiconference. pp. 716-722.

Prepared using sagej.cls 
[12] Barros FJ. Dynamic structure discrete event system specification: a new formalism for dynamic structure modeling and simulation. In Proceedings of the 1995 Winter Simulation Multiconference. pp. 781-785.

[13] Chow ACH, Zeigler BP and Kim DH. Abstract simulator for the parallel DEVS formalism. In AI, Simulation, and Planning in High Autonomy Systems. pp. 157-163.

[14] Himmelspach J and Uhrmacher AM. Sequential processing of PDEVS models. In Proceedings of the 3rd European Modeling 83 Simulation Symposium. pp. 239-244.

[15] Barros FJ. Modeling formalisms for dynamic structure systems. ACM Transactions on Modeling and Computer Simulation 1997; 7: 501-515.

[16] Barros FJ. Abstract simulators for the DSDE formalism. In Proceedings of the 1998 Winter simulation Multiconference. pp. 407-412.

[17] Uhrmacher AM. Dynamic structures in modeling and simulation: a reflective approach. ACM Transactions on Modeling and Computer Simulation 2001; 11: 206-232.

[18] Wainer G and Giambiasi N. Discrete event modeling and simulation technologies. chapter Timed cell-DEVS: modeling and simulation of cell spaces. Springer-Verlag New York, Inc., 2001. pp. 187-214.

[19] Troccoli A and Wainer G. Implementing Parallel Cell-DEVS. In Proceedings of the 2003 Spring Simulation Symposium. pp. 273-280.

[20] Nutaro JJ. adevs. http://www.ornl.gov/ 1qn/adevs/, 2015.

[21] Muzy A and Nutaro JJ. Algorithms for efficient implementations of the DEVS \& DSDEVS abstract simulators. In 1st Open International Conference on Modeling and Simulation (OICMS). pp. 273-279.

[22] Van Tendeloo Y and Vangheluwe H. The modular architecture of the Python(P)DEVS simulation kernel. In Proceedings of the 2014 Spring Simulation Multiconference. pp. 387-392.

[23] Fritzson P, Aronsson P, Lundvall H et al. The OpenModelica Modeling, Simulation, and Software Development Environment. Simulation News Europe 2005; 44: 8-16.

[24] Wainer G. CD++: a toolkit to develop DEVS models. Software: Practice and Experience 2002; 32(13): 1261-1306.

[25] Muzy A and Wainer G. Comparing simulation methods for fire spreading across a fuel bed. In Proceedings of AIS'2002. pp. 219-224.

Prepared using sagej.cls 
[26] Shang H and Wainer G. A model of virus spreading using Cell-DEVS. In Computational Science ICCS 2005, Lecture Notes in Computer Science, volume 3515. Springer Berlin / Heidelberg, 2005. pp. 145-201.

[27] Wainer G and Giambiasi N. Application of the Cell-DEVS Paradigm for Cell Spaces Modelling and Simulation. SIMULATION 2001; 76(1): 22-39.

[28] Kgwadi M, Shang H and Wainer G. Definition of dynamic DEVS models: Dynamic Structure CD++. In Proceedings of the 2008 Spring Simulation Multiconference. pp. 10:1-10:4.

[29] Kim S, Sarjoughian HS and Elamvazhuthi V. DEVS-Suite: a simulator supporting visual experimentation design and behavior monitoring. In Proceedings of the 2009 Spring Simulation Multiconference. pp. 161:1161:7.

[30] Sarjoughian $\mathrm{H}$ and Zeigler B. DEVSJava: Basis for a DEVS-based Collaborative M\&S Environment. SIMULATION 1998; 30: 29-36.

[31] Chezzi CM, Tymoschuk AR and Lerman R. A Method for DEVS Simulation of e-Commerce Processes for Integrated Business and Technology Evaluation (WIP). In Proceedings of the 2013 Spring Simulation Multiconference. pp. 13:1-13:6.

[32] Palaniappan S, Sawhney A and Sarjoughian HS. Application of the DEVS Framework in Construction Simulation. In Proceedings of the 38th Conference on Winter Simulation. Winter Simulation Conference, pp. 2077-2086.

[33] Ferayorni AE and Sarjoughian HS. Domain driven simulation modeling for software design. In Proceedings of the 2007 Summer Computer Simulation Conference. pp. 297-304.

[34] Seo C, Zeigler BP, Coop R et al. DEVS modeling and simulation methodology with MS4Me software. In Proceedings of the 2013 Spring Simulation Multiconference. pp. 33:1-33:8.

[35] Kim T, Lee C, Christensen E et al. System entity structuring and model base management. IEEE Transactions on Systems, Man and Cybernetics 1990; 20(5): 1013-1024.

[36] Zeigler BP, Seo C, Coop R et al. Creating Suites of Models with System Entity Structure: Global Warming Example. In Proceedings of the 2013 Spring Simulation Multiconference. pp. 32:1-32:8.

[37] Zeigler B, Seo C and Kim D. System entity structures for suites of simulation models. International Journal of Modeling, Simulation, and Scientific Computing 2013; 4: 3:1-3:11.

Prepared using sagej.cls 
[38] Bergero F and Kofman E. PowerDEVS: a tool for hybrid system modeling and real-time simulation. Simulation 2011; 87: 113-132.

[39] Kofman E, Lapadula M and Pagliero E. PowerDEVS: A DEVS-Based Environment for Hybrid System Modeling and Simulation. Technical report, School of Electronic Engineering, Universidad Nacional de Rosario, 2003.

[40] Capocchi L, Santucci JF, Poggi B et al. DEVSimPy: A collaborative python software for modeling and simulation of DEVS systems. In Workshop on Enabling Technologies: Infrastructure for Collaborative Enterprises. pp. 170-175.

[41] Van Mierlo S, Van Tendeloo Y, Barroca B et al. Explicit Modelling of a Parallel DEVS Experimentation Environment. In Proceedings of the 2015 Spring Simulation Multiconference. pp. 860-867.

[42] Van Tendeloo $\mathrm{Y}$ and Vangheluwe H. PythonPDEVS: a distributed Parallel DEVS simulator. In Proceedings of the 2015 Spring Simulation Multiconference. pp. 844-851.

[43] Quesnel G, Duboz R, Ramat E et al. VLE: a multimodeling and simulation environment. In Proceedings of the 2007 Summer Simulation Multiconference. pp. 367-374.

[44] Hwang MH. X-S-Y. https://code.google.com/p/x-s-y/, 2012.

[45] Tewoldeberhan TW, Verbraeck A, Valentin E et al. An evaluation and selection methodology for discrete-event simulation software. In Proceedings of the 2002 Winter Simulation Multiconference. pp. 67-75.

[46] Fujimoto RM. Parallel and Distribution Simulation Systems. 1st ed. New York, NY, USA: John Wiley \& Sons, Inc., 1999.

[47] Glinsky E and Wainer G. Definition of Real-Time Simulation in the CD++ Toolkit. In Proceedings of the 2002 Summer Simulation Multiconference.

[48] Martin D, McBrayer T, Radhakrishan R et al. Time warp parallel discrete event simulator. Technical report, Computer Architecture Design Laboratory. University of Cincinnati. USA, 1997.

[49] Barroca B, Mustafiz S, Van Mierlo S et al. Integrating a Neutral Action Language in a DEVS Modelling Environment. In Proceedings of the 8th International ICST Conference on Simulation Tools and Techniques. pp. $19-28$.

[50] Sun Y and Hu X. Partial-modular DEVS for improving performance of cellular space wildfire spread simulation. In Proceedings of the 2008 Winter Simulation Multiconference. pp. 1038-1046.

Prepared using sagej.cls 
[51] Posse E. Modelling and simulation of dynamic structure discrete-event systems. PhD Thesis, School of Computer Science, McGill University, 2008.

[52] Ousterhout JK. Scripting: Higher-Level Programming for the 21st Century. Computer 1998; 31(3): 23-30.

[53] Chen B and Vangheluwe H. Symbolic flattening of DEVS models. In Proceedings of the 2010 Summer Simulation Multiconference. pp. 209-218.

[54] Van Tendeloo Y and Vangheluwe H. Activity in PythonPDEVS. In Proceedings of ACTIMS 2014. pp. 2:1-2:10.

\section{Author Biographies}

Yentl Van Tendeloo is a PhD student at the University of Antwerp, Department of Mathematics and Computer Science, Antwerp, Belgium.

Hans Vangheluwe is a full professor at the University of Antwerp, Department of Mathematics and Computer Science, Antwerp, Belgium. He is also an adjunct professor at McGill University, School of Computer Science, Montréal, Canada, where he was a full professor before.

Prepared using sagej.cls 